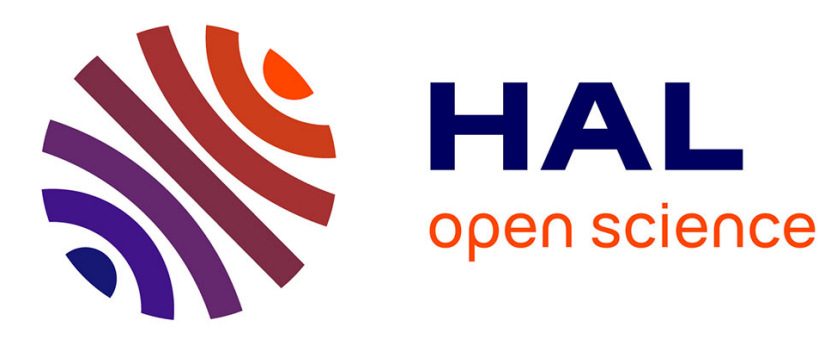

\title{
Computing Beltrami Fields
}

Tahar Amari, Cédric Boulbe, Tahar Zamène Boulmezaoud

\section{To cite this version:}

Tahar Amari, Cédric Boulbe, Tahar Zamène Boulmezaoud. Computing Beltrami Fields. SIAM Journal on Scientific Computing, 2009, 31 (5), pp. 3217-3254. hal-00414389

\section{HAL Id: hal-00414389 \\ https://hal.science/hal-00414389}

Submitted on 26 Nov 2009

HAL is a multi-disciplinary open access archive for the deposit and dissemination of scientific research documents, whether they are published or not. The documents may come from teaching and research institutions in France or abroad, or from public or private research centers.
L'archive ouverte pluridisciplinaire HAL, est destinée au dépôt et à la diffusion de documents scientifiques de niveau recherche, publiés ou non, émanant des établissements d'enseignement et de recherche français ou étrangers, des laboratoires publics ou privés. 


\title{
COMPUTING BELTRAMI FIELDS
}

TAHAR AMARI*, CÉDRIC BOULBE ${ }^{\dagger}$, AND TAHAR ZAMÈNE BOULMEZAOUD ${ }^{\ddagger}$

\begin{abstract}
For solving the non-linear equations governing force-free fields, an iterative methodology based on the splitting of the problem is described. On the basis of this splitting, three families of subproblems have to be solved numerically. The first problem consists to find a potential field. A mixed hybrid method is used to solve it. The second problem, which is a curl-div system, is solved by means of a mixed method. The last problem is a transport equation which is approximated using a streamline diffusion technique. Numerical 3D experiences and results are given to illustrate the efficiency of the method.
\end{abstract}

Key words. Force-free fields, Beltrami fields, Steady Euler equations, ABC flows, fusion.

AMS subject classifications. 35F30, 35M10, 35A35, 35Q35, 76B03, 85-08, 85A30.

1. Introduction. A three-dimensional vector field $\boldsymbol{B}$ is said Beltrami if

$$
\operatorname{curl} B \times B=0
$$

and

$$
\operatorname{div} \boldsymbol{B}=0
$$

The non-linear equation (1.1) means that $\boldsymbol{B}$ and its curl are everywhere colinear. In astrophysics and in plasma fusion Beltrami fields are known as force-free fields. They describe the equilibrium of a perfectly conducting pressureless plasma in the presence of a strong magnetic field. In that case, $\boldsymbol{B}$ corresponds to the magnetic field, while $\boldsymbol{j}=\operatorname{curl} \boldsymbol{B}$ is nothing but the current density. Equation (1.1) means that the only significant force acting on the plasma, say the Lorentz force, vanishes in equilibrium. It corresponds to the pressureless $(p=0)$ case of the magnetostatic equations

$$
\begin{aligned}
\boldsymbol{j} \times \boldsymbol{B} & =\nabla p \\
\boldsymbol{j} & =\operatorname{curl} \boldsymbol{B}, \\
\operatorname{div} \boldsymbol{B} & =0
\end{aligned}
$$

The magnetostatic equations appears for example in magnetized thermonuclear fusion in toroidal geometries. They describe the balance between the magnetic pressure and the kinematic pressure of a magnetized confined plasma.

In the astrophysical context, Beltrami fields are often encountered to describe magnetic models of outer atmospheres above accretion disks or the low solar corona. It is indeed well known that a large part of the solar atmosphere (low corona below three solar radius) is dominated by the magnetic field which is created inside the sun by a dynamo process, and then emerges in the above atmosphere. This magnetic field is responsible for most of the structures and phenomena observed in various wavelengths in the corona such as : prominences, small scale and large scale eruptive

\footnotetext{
*Centre de Physique Théorique, École Polytechnique, 91128 Palaiseau Cedex, France (tahar.amari@cpht.polytechnique.fr).

${ }^{\dagger}$ Laboratoire J. A. Dieudonné, Université de Nice, Faculté des Sciences, Parc Valrose, 06108 Nice Cedex 02 (cedric.boulbe@unice.fr)

$\ddagger$ Laboratoire de Mathématiques, Université de Versailles Saint-Quentin-en-Yvelines, Bâtiment Fermat, 45 avenue des Etats-Unis, 78035 Versailles, France (boulmezaoud@math.uvsq.fr).
} 
events which may release large amount of energy and whose consequences may propagate up to the earth environment, as well as probably heating of the solar corona. Unfortunately, the magnetic field is difficult to measure locally in the hot and tenuous corona, whereas it is important to understand its three dimensional properties to elaborate models of such structures and phenomena, at the basis of any theoretical model. However, the magnetic field is measurable in the cooler and denser plasma that represent the "surface" of the sun (called the photosphere). Therefore astrophysicists have developed an approach which consists in Reconstructing the three-dimensional coronal magnetic field above the photosphere, from boundary data given on the photosphere. The problem then consists in solving equations (1.1-1.2) for given boundary conditions. As we will discuss later, although the three components of the magnetic fields are known on the sun surface, in order to have a well set problem for the mixed elliptic-hyperbolic problem above, only part of this information can be imposed on the boundary. The reader can refer to $[2,17]$ and references therein for more details about Beltrami fields in solar physics.

In fluid dynamics, experience shows that flows in which vorticity is roughly aligned to velocity, as the case of a Beltrami fields, could develop some instabilities. Moreover, Beltrami flows are solutions of the steady Euler equations describing a 3D steady inviscid and incompressible flows

$$
\boldsymbol{u} . \nabla \boldsymbol{u}+\nabla p=\mathbf{f}, \operatorname{div} \boldsymbol{u}=0 \text { in } \Omega .
$$

Indeed, the last system can be written as

$$
\operatorname{curl} \boldsymbol{u} \times \boldsymbol{u}+\nabla\left(p+\frac{|\boldsymbol{u}|^{2}}{2}\right)=\boldsymbol{f}, \operatorname{div} \boldsymbol{u}=0 \text { in } \Omega .
$$

Hence, if $\boldsymbol{B}$ satisfies $(1.1)+(1.2)$, then the pair $(\boldsymbol{B}, p)$ with $p(x)=-\frac{|\boldsymbol{B}|^{2}}{2}$ is obviously solution to (1.3) provided that $\mathbf{f}=0$.

It is worth noting that Beltrami 3D fields are also linked to two dimensional steady Euler' equations in a different and surprising manner. More precisely, to each solution

$$
\left(\left(u_{1}(x, y), u_{2}(x, y)\right), p(x, y)\right)
$$

of the 2D Euler's equations (1.3), one can associate a 3D Beltrami field $\boldsymbol{B}=\left(v_{1}, v_{2}, v_{3}\right)$ defined as

$$
\begin{aligned}
& v_{1}(x, y)=u_{1}(x, y) \\
& v_{2}(x, y)=u_{2}(x, y) \\
& v_{3}(x, y)=\psi(x, y)
\end{aligned}
$$

where $\psi(x, y)$ is chosen such as

$$
\psi^{2}(x, y)+2 p(x, y)+u_{1}^{2}(x, y)+u_{2}^{2}(x, y)=\text { constant } .
$$

In that case, we have

$$
\operatorname{curl} \boldsymbol{B}=\frac{\omega(x, y)}{\psi(x, y)} \boldsymbol{B}
$$


where $\omega(x, y)$ is the $2 \mathrm{D}$ vorticity defined by

$$
\omega(x, y)=\frac{\partial u_{2}}{\partial x}-\frac{\partial u_{1}}{\partial y} .
$$

The interested reader can refer to Bruno and Laurence [22] and Alber [1] for the proof of existence of smooth solutions to 3D steady Euler's equations (see also Glass [29] for some consideration concerning the two dimensional case).

From a mathematical viewpoint, equation (1.1) can be roughly rewritten in the form

$$
\operatorname{curl} \boldsymbol{B}=\lambda(\boldsymbol{x}) \boldsymbol{B}
$$

where $\lambda(\boldsymbol{x})$ is a scalar function which varies in general with position. It turned out to be more convenient to work with (1.5) than (1.1). A common way to study (1.5) is to distinguish three categories of Beltrami' flows.

- Potential fields. It corresponds to $\lambda \equiv 0$. In that case, $\boldsymbol{B}$ is irrotational and derives from a potential. Namely, $\boldsymbol{B}$ is of the form $\boldsymbol{B}=\nabla \varphi$ with $\Delta \varphi=0$ (when $\Omega$ is simply-connected). This is the simplest and most popular model.

- Linear Beltrami fields: they are characterized by a constant $\lambda$. The existence of linear force-free fields when $\lambda$ and $\boldsymbol{B} . \boldsymbol{n}_{\mid \partial \Omega}$ are prescribed was treated in the literature in several geometries (simply or multiply connected bounded or unbounded domains). The reader can refer to [36, 37], [38], [17] and references therein.

- Non-linear Beltrami fields: they corresponds to a variable function $\lambda($.$) . It$ is much less obvious whether non-linear Beltrami field with prescribed data on the boundary of $\Omega$ exists. Bineau [13] gives a proof of the existence of non-linear force-free fields under some restrictive assumptions including the requierements that the vector field is regular and has a simple topology and that $\lambda$ is small. In [15] Boulmezaoud and Amari have proven under few assumptions the existence of $H^{1}$ Beltrami fields with $\lambda \in L^{\infty}(\Omega)$ and in bounded but not necessarily simply-connected domains (see also [14] and Section 2 hereafter).

There are several explicit examples in the literature of linear Beltrami fields. Among these examples, let us quote the celebrated ABC flows (after V. Arnold, E. Beltrami and S. Childress) and for which Arnold [7], and independently Childress [23], conjuctured the existence of chaotic paths. ABC flows were studied by Dombre and al. [26] and are today a classical example of 3D chaotic phenomena and lagrangian turbulence (see also Hénon [33]). They are defined in the cartezian coordinates as follows

$$
\begin{aligned}
& u_{1}=A \sin z+C \cos y, \\
& u_{2}=B \sin x+A \cos z, \\
& u_{3}=C \sin y+B \cos x .
\end{aligned}
$$

On the contrary, there are few analytical examples of Beltrami fields are available with a non-constant proportionality factor $\lambda$. Examples of Beltrami fields in cylindrical geometry can be found in $[45,46]$. In spherical geometry, the reader can refer to Low (1982-1988) for an explicit Beltrami flow. Without breaking down the corresponding calculus, we give here two great families of non-linear Beltrami fields.

Example 1 (spherical force-free fields). Let $f: \mathbb{C} \rightarrow \mathbb{C}$ be an holomorphic function 
and let $\chi:] 0,+\infty\left[\rightarrow \mathbb{R}\right.$ be a real function of class $\mathscr{C}^{1}$. Let $(r=|\boldsymbol{x}|, \theta, \varphi)$ be the classical spherical coordinates and set

$$
\xi=\ln \tan \frac{\theta}{2}+i \varphi, \Psi(\boldsymbol{x})=\operatorname{Re}\left(f(\xi) e^{i \chi(|\boldsymbol{x}|)}\right)
$$

Then, the vector field

$$
\boldsymbol{B}=\frac{\boldsymbol{x}}{|\boldsymbol{x}|} \times \nabla \Psi
$$

is Beltrami and satisfies

$$
\operatorname{curl} B=-\chi^{\prime}(|x|) B
$$

Observe that

$$
\boldsymbol{x} . \boldsymbol{B}=\mathbf{0} .
$$

We call these fields "spherical force-free fields" since their fieldlines are inscribed on the spheres $|\boldsymbol{x}|=$ constant. Notice that the well known solution due to Low [41] corresponds to particular case $f(z)=-i z$.

Example 2 (cylindrical force-free fields). Let $(\rho, \theta, z)$ be the cylindrical coordinates and let $\eta$ a positive real function such that $0 \leq \eta^{\prime}(t) \leq 1$ for each $t \in \mathbb{R}$. Then, the vector field

$$
\boldsymbol{B}=A e^{-\eta(\ln \rho)}\left(\sqrt{\eta^{\prime}(\ln \rho)} \boldsymbol{e}_{\theta} \pm \sqrt{1-\eta^{\prime}(\ln (\rho))} \boldsymbol{e}_{z}\right)
$$

is Beltrami and satisfies

$$
\operatorname{curl} \boldsymbol{B}= \pm \frac{1}{\rho} \frac{2 \eta^{\prime}(\ln \rho)+\eta^{\prime \prime}(\ln \rho)-2 \eta^{\prime}(\ln \rho)^{2}}{2 \sqrt{\eta^{\prime}(\ln \rho)} \sqrt{1-\eta^{\prime}(\ln \rho)}} \boldsymbol{B} .
$$

The fieldlines of such cylindrical force-free fields are inscribed on cylinders $\rho=$ constant.

Computation of non-linear Beltrami fields is among the most difficult stages in their studying. Existing methods proposed for solving the problem (1.5) $+(1.2)$ can be distinguished mainly into four kinds: MHD or CFD relaxation methods, vertical integration methods and iterative methods. The idea of MHD relaxation approaches consists to start with an adequate vector field and to relax it into a force-free state by means of magnetohydrodynamic equations (see, e.g., Priest [46] and Friedberg [27]). Among CFD relaxation methods one can notice the artificial compression method which was proposed by Chorin [25] and improved by some other authors (see [40] for its use in computing Beltrami fields). Vertical integration methods consists to rewrite the problem $(1.5)+(1.2)$ as an evolution problem in the $z$-direction and then integrating in the direction of increasing $z$ (see [3]). Iteratives method are often based on a fixed point scheme.

The purpose of this paper is to propose an iterative algorithm and a finite element method for computing Beltrami flows. The emphasis is put on the accuracy of the approximation and also on some properties of Beltrami fields, like their energy and their helicity.

The remaining of this paper is organized as follows 
- Section 2 is devoted to a review of the functional framework we use here. We recall some results concerning the existence of linear and non-linear Beltrami flows.

- In Section 3 we expose a fixed-point strategy for computing Beltrami flows in bounded domains.

- In Section 4, we deal with discretization of the three subproblems involved in the fixed point algorithm. An appropriate finite element method is used for solving each one of these problems. After exposing the features of the method, we analyze carefully its convergence.

- In Section 5, we present some 3D computational results obtained by a 3D code based on the iterative algorithm exposed in Section 2 and on the discretization methods developed in Section 4. Some global properties (energy, helicity,...etc) are emphasized by the way.

2. Notations. Mathematical statement of the problem. Let $\Omega$ be a bounded open set of $\mathbb{R}^{3}$, not necessarily simply connected, with a $\mathscr{C}^{1,1}$ boundary or is convex polyhedron. We shall denote by $\Gamma_{0}, \ldots, \Gamma_{p}$ the components of $\partial \Omega$, with $\Gamma_{0}$ the exterior boundary of $\Omega$, say the boundary of the unbounded component of $\mathbb{R}^{3} \backslash \bar{\Omega}$.

If $\Omega$ is not simply-connected (for example if $\Omega$ is a torus), then we assume that there exists a $m$ manifolds $(m \geq 1)$ of dimension $2, \Sigma_{1}, \ldots, \Sigma_{m}$, such that

- $\Omega=\Omega_{0} \backslash \cup_{i=1}^{m} \Sigma_{i}$ is smooth and simply-connected,

- $\forall i, j \leq m, \Sigma_{i} \cap \Sigma_{j}=\emptyset$ if $i \neq j$,

- $\forall i \leq m, \partial \Sigma_{i} \subset \partial \Omega$.

The integer $m$ describes the connectedness of $\Omega$. When $\Omega$ is simply-connected, we set $m=0$. When $\Omega$ is the torus, $m=1$ and $\Sigma_{1}$ can be chosen as a cross-section (see figure 2.1).

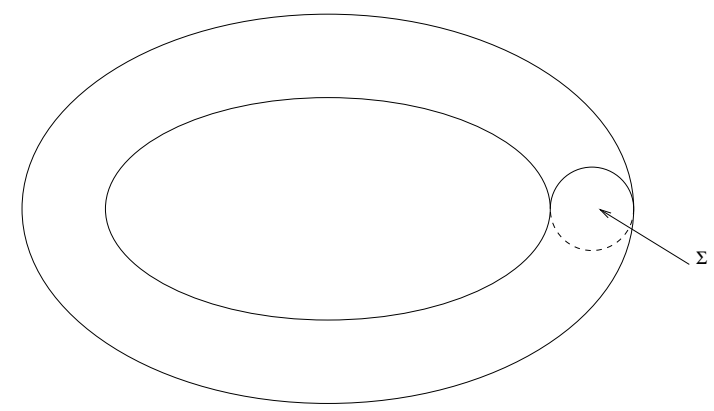

FIG. 2.1. The three-dimensional torus with a cross-section $\Sigma$

In the sequel, we use bold characters for vector functions or distributions. For any $k \in \mathbb{N}, \mathbb{P}_{k}$ stands for the space of polynomial of degree lower than $k$, and $\mathbb{H}_{k}$ is the space of the homogeneous polynomials of degree equal to $k$. If $k$ is a non positive integer, we set by convention $\mathbb{P}_{k}=\mathbb{H}_{k}=\{0\}$. Let $\mathscr{D}(\Omega)$ be the well-known space of $\mathscr{C}^{\infty}$ functions with a compact support on $\Omega$, and let $\mathscr{D}^{\prime}(\Omega)$ be its dual space, namely the space of distributions. We denote by $\langle. .$.$\rangle the duality. For 1 \leq p \leq+\infty, L^{p}(\Omega)$ stands for the space of (equivalence classes of) all measurable functions that are $p^{\text {th }}$ power integrable on $\Omega$. This space is equipped with its usual norm $\|u\|_{L^{p}(\Omega)}=\left(\int_{\Omega}|u|^{p} d x\right)^{1 / p}$ (with the usual modification when $p=+\infty)$. For each $v \in L^{1}(\Omega)$, we write $\bar{v}=\frac{1}{|\Omega|} \int_{\Omega} v(x) d x$, and we denote by $L_{0}^{2}(\Omega)$ the subspace of $L^{2}(\Omega)$ composed of those functions $v \in L^{2}(\Omega)$ 
satisfying $\bar{v}=0$.

Given an integer $m \geq 0, H^{m}(\Omega)$ refers to classical Sobolev space of all the functions $u$ whose generalized derivatives satisfy: $\forall \mu \in \mathbb{N}^{3} ; D^{\mu} u \in L^{2}(\Omega)$. This space is equipped with the norm $\|v\|_{m}=\left(\sum_{|\mu| \leq m}\left\|D^{\mu} u\right\|_{0, \Omega}^{2}\right)^{1 / 2}$. We define also the spaces

$$
\begin{aligned}
H(\operatorname{curl} ; \Omega) & =\left\{\boldsymbol{v} \in L^{2}(\Omega)^{3} \mid \operatorname{curl} \boldsymbol{v} \in L^{2}(\Omega)^{3}\right\} \\
H(\operatorname{div} ; \Omega) & =\left\{\boldsymbol{v} \in L^{2}(\Omega)^{3} \mid \operatorname{div} \boldsymbol{v} \in L^{2}(\Omega)\right\}
\end{aligned}
$$

equipped respectively with the norms

$$
\begin{gathered}
\|\boldsymbol{v}\|_{H(\operatorname{curl} ; \Omega)}=\left(\|\boldsymbol{v}\|_{0, \Omega}^{2}+\|\operatorname{curl} \boldsymbol{v}\|_{0, \Omega}^{2}\right)^{1 / 2}, \\
\|\boldsymbol{v}\|_{H(\operatorname{div} ; \Omega)}=\left(\|\boldsymbol{v}\|_{0, \Omega}^{2}+\|\operatorname{div} \boldsymbol{v}\|_{0, \Omega}^{2}\right)^{1 / 2} .
\end{gathered}
$$

Finally, consider the space

$$
U=\{\boldsymbol{v} \in H(\operatorname{div} ; \Omega) \cap H(\operatorname{curl} ; \Omega) \mid \boldsymbol{v} . \boldsymbol{n}=0 \text { on } \partial \Omega\},
$$

equipped with the norm :

$$
\|\boldsymbol{u}\|_{U}=\left(\|\boldsymbol{u}\|_{0, \Omega}^{2}+\|\operatorname{div} \boldsymbol{u}\|_{0, \Omega}^{2}+\|\operatorname{curl} \boldsymbol{u}\|_{0, \Omega}^{2}\right)^{1 / 2} .
$$

Notice that

$$
U \hookrightarrow H^{1}(\Omega)^{3}
$$

since $\Omega$ has a $\mathscr{C}^{1,1}$ - boundary or is a convex polyhedron (see, e. g., [28] and references therein).

It is well known that the subspace

$$
H=\{\boldsymbol{u} \in U \mid \operatorname{curl} \boldsymbol{u}=\mathbf{0} \text { and } \operatorname{div} \boldsymbol{u}=0 \text { in } \Omega\},
$$

is finite dimensional and $\operatorname{dim} H=m$. Moreover, the semi-norm

$$
\boldsymbol{u} \rightarrow|\boldsymbol{u}|_{U}=\left(\|\operatorname{div} \boldsymbol{u}\|_{0, \Omega}^{2}+\|\operatorname{curl} \boldsymbol{u}\|_{0, \Omega}^{2}+\left\|\mathbb{P}_{H} \boldsymbol{u}\right\|^{2}\right)^{1 / 2}
$$

is a norm on $U$, equivalent to the norm $\|.\|_{U}$ (see, e. g., [28] and references therein). Here $\mathbb{P}_{H}$ denotes the orthogonal projection form $U$ on $H$. If $\Omega$ is simply-connected, then $H=\{\mathbf{0}\}$ and $\mathbb{P}_{H}=0$.

In the sequel, we set

$$
\mu_{0}(\Omega)=\inf _{\boldsymbol{u} \in U, \boldsymbol{u} \neq 0} \frac{|\boldsymbol{u}|_{U}}{\|\boldsymbol{u}\|_{0, \Omega}}>0 .
$$

We have the following estimate (see [17])

$$
\mu_{0}^{2} \leq \lambda_{1, N},
$$

where $\lambda_{1, N}$ is the first non-vanishing eigenvalue of $-\Delta$ with an homogeneous Neumann condition. 
Now, let us consider the system

$$
\operatorname{curl} \boldsymbol{B}=\lambda(\boldsymbol{x}) \boldsymbol{B}, \operatorname{div} \boldsymbol{B}=0 .
$$

This system of equations must be completed with some boundary conditions on the vector field $\boldsymbol{B}$ and on the scalar function $\lambda(\boldsymbol{x})$. As for potential fields (for which $\lambda$ vanishes everywhere), we prescribe the normal component of $\boldsymbol{B}$ on whole the boundary $\partial \Omega$

$$
\boldsymbol{B} . \boldsymbol{n}=g \text { on } \partial \Omega .
$$

When the domain is multiply-connected ( $m \geq 1$ ), the flux of $\boldsymbol{B}$ throughout the cuts $\Sigma_{i}, 1 \leq i \leq p$, is also prescribed

$$
\int_{\Sigma_{i}} \boldsymbol{B} . \boldsymbol{n}=a_{i}, \forall i \in\{1, \ldots, m\} .
$$

The function $g$ in (2.3) must satisfy the compatibility condition

$$
\int_{\partial \Omega} g d \sigma=0
$$

since $\boldsymbol{B}$ is divergence free in $\Omega$.

To see what boundary conditions should be imposed on $\lambda$, observe that applying the divergence operator to $(2.2)$ yields

$$
B \cdot \nabla \lambda=0 .
$$

Equation (2.6) can be viewed as a first order hyperbolic equation the characteristics of which are the field lines of $\boldsymbol{B}$ (see Section 3 hereafter). In other words, (2.6) means that $\lambda$ is constant along those fieldlines. Hence, it is a natural idea to prescribe $\lambda$ on the inflow boundary, namely

$$
\lambda=\alpha_{0} \text { on } \Sigma^{-},
$$

where

$$
\Sigma^{-}=\{\boldsymbol{x} \in \partial \Omega ; \boldsymbol{B}(x) \cdot \boldsymbol{n}(x)=g(\boldsymbol{x})<0\} .
$$

Condition (2.7) can also be viewed as a boundary condition on the curl of $\boldsymbol{B}$, that is

$$
\operatorname{curl} \boldsymbol{B} . \boldsymbol{n}=h \text { on } \Sigma^{-},
$$

with $h(\boldsymbol{x})=\alpha_{0}(\boldsymbol{x}) g(\boldsymbol{x})$.

When $\Omega$ is multiply-connected ( $m \geq 1$ ), it could be useful to add another condition on fluxes of $\operatorname{curl} \boldsymbol{B}$ throughout the cuts $\Sigma_{i}, 1 \leq i \leq p$,

$$
\int_{\Sigma_{i}} \operatorname{curl} \boldsymbol{B} . \boldsymbol{n}=b_{i}, \forall i \in\{1, \ldots, m\} .
$$

REMARK 2.1. In the case of a linear force-free field (see, e. g., [17]), a constant value of $\lambda$ (or the helicity) is given and conditions (2.7) and (2.9) are dropped. 
In the following, $\boldsymbol{B}_{0}$ will be the irrotational field having the same boundary conditions as $\boldsymbol{B}$, that is, $\boldsymbol{B}_{0}$ is the unique vector field in $H^{1}(\Omega)^{2}$ satisfying

$$
\operatorname{curl} \boldsymbol{B}_{0}=0 \text { in } \Omega, \operatorname{div} \boldsymbol{B}_{0}=0 \text { in } \Omega, \boldsymbol{B}_{0} \cdot \boldsymbol{n}=g \text { on } \partial \Omega,
$$

and, when $\Omega$ is multiply-connected,

$$
\int_{\Sigma_{i}} \boldsymbol{B}_{0} \cdot \boldsymbol{n}=a_{i}, \forall i \in\{1, \ldots, m\}
$$

When $\Omega$ is simply-connected, $\boldsymbol{B}_{0}$ is of the form $\boldsymbol{B}_{0}=\nabla \varphi$, with $\varphi$ solution of the Neumann problem

$$
\Delta \varphi=0, \text { and } \frac{\partial \varphi}{\partial n}=g \text { on } \partial \Omega
$$

When $\Omega$ is multiply-connected, $\boldsymbol{B}_{0}$ writes $\boldsymbol{B}_{0}=\nabla \varphi+\boldsymbol{h}$, with $\boldsymbol{h}$ an element of $H$ (see [17] for more details).

At this stage, two situations are distinguished

\section{(A) Linear Beltrami fields}

In this case, $\lambda$ is a known constant. The boundary condition (2.7) is dropped since $\lambda$ is given and one can consider the typical problem:

$$
\left\{\begin{array}{lll}
\text { Find } \boldsymbol{B} \text { such as } & & \\
\operatorname{curl} \boldsymbol{B} & =\lambda \boldsymbol{B} & \text { in } \Omega, \\
\operatorname{div} \boldsymbol{B} & =0 & \text { in } \Omega, \\
\boldsymbol{B} . \boldsymbol{n} & =g, & \text { on } \partial \Omega \\
\int_{\Sigma_{i}} \boldsymbol{B} \cdot \boldsymbol{n} d \sigma & =a_{i}, \forall i \in\{1, \ldots, m\} .
\end{array}\right.
$$

When $\lambda \neq 0$, the boundary function $g$ must satisfy the compatibility conditions (see Lemma 4.5 hereafter)

$$
\int_{\Gamma_{i}} g d \sigma=0, \text { for } 0 \leq i \leq p .
$$

Problem (2.12) was treated in [17]. The following result is stated

THEOREM 2.1. There exists a countable sequence of reals $\left(\mu_{i}\right)_{i \geq \mathbb{N}}$ satisfying

- $\left|\mu_{i}\right| \geq \mu_{0}$ for each $i \in \mathbb{N}$,

- $\lim _{i \mapsto+\infty}\left|\mu_{i}\right|=+\infty$,

and such that

- If $\lambda \notin\left\{\mu_{0}, \mu_{1}, \ldots\right\}$, then the problem (2.12) admits one and only one solution $\boldsymbol{B} \in H^{1}(\Omega)^{3}$ for each $g \in H^{1 / 2}(\partial \Omega)$ satisfying (2.13).

- If $\lambda \in\left\{\mu_{0}, \mu_{1}, \ldots\right\}$, the problem (2.12) has at least one solution if and only if $g$ satisfies some additional compatibility conditions (see [17]). In that case, the solution is unique, up to elements of a finite dimensional space.

Moreover, if $|\lambda|<\mu_{0}$, then

$$
\left\|\boldsymbol{B}_{0}\right\|_{0, \Omega}^{2} \leq\|\boldsymbol{B}\|_{0, \Omega}^{2} \leq \frac{\mu_{0}^{2}}{\mu_{0}^{2}-\lambda^{2}}\left\|\boldsymbol{B}_{0}\right\|_{0, \Omega}^{2} .
$$




\section{(B) Non-linear Beltrami fields}

As underlined before, non-linear Beltrami fields correspond to a variable function $\lambda$. The boundary value problem we consider is

$$
\left\{\begin{array}{rlrl}
\text { Find a pair }(\boldsymbol{B}, \lambda) & \text { such as: } \\
\operatorname{curl} \boldsymbol{B} & =\lambda(\boldsymbol{x}) \boldsymbol{B} & & \text { in } \Omega, \\
\operatorname{div} \boldsymbol{B} & =0 & & \text { in } \Omega, \\
\boldsymbol{B} \cdot \boldsymbol{n} & =g & & \text { on } \partial \Omega, \\
\lambda & =\alpha_{0} & & \text { on } \Sigma^{-} .
\end{array}\right.
$$

This BVP is completed with conditions (2.4) and (2.9) when the domain is multiply-connected. In [15] and [14] the authors proved the following existence results in a regular bounded domain with a connected boundary $(p=0)$ Theorem 2.2. Assume that $g$ is of class $\mathscr{C}^{1}$ and that $\alpha_{0} \in L^{\infty}\left(\Sigma^{-}\right)$with

$$
\text { supess }_{\boldsymbol{x} \in \Sigma^{-}}\left|\alpha_{0}(\boldsymbol{x})\right|=\left\|\alpha_{0}\right\|_{\infty, \Sigma^{-}}<\mu_{0} .
$$

Then the problem (2.15) has at least one solution $(\boldsymbol{B}, \lambda) \in H^{1}(\Omega)^{3} \times L^{\infty}(\Omega)$. This solution satisfies the estimate

$$
\begin{gathered}
\|\lambda\|_{\infty} \leq\left\|\alpha_{0}\right\|_{\infty, \Sigma^{-}}, \\
E_{0} \leq\|\boldsymbol{B}\|_{0, \Omega}^{2} \leq \tau E_{0} .
\end{gathered}
$$

where $E_{0}=\left\|\boldsymbol{B}_{0}\right\|_{0, \Omega}^{2}$ and $\tau=1+\frac{\left\|\alpha_{0}\right\|_{\infty, \Sigma^{-}}^{2}}{\left(\mu_{0}-\left\|\alpha_{0}\right\|_{\infty, \Sigma^{-}}\right)^{2}}$.

Theorem 2.3. Let $g \in H^{1 / 2}(\partial \Omega)$ and $\alpha_{0} \in H^{3 / 2}\left(\Sigma^{-}\right) \cap L^{\infty}\left(\Sigma^{-}\right)$with $\alpha_{0}$ satisfying (2.16). Then, the problem (2.15) has at least one solution $(\boldsymbol{B}, \lambda) \in$ $H^{1}(\Omega)^{3} \times L^{\infty}(\Omega)$. Moreover, this solution satisfies also the estimates (2.17) and (2.18). The existence of non-linear force-free fields domains with a non connected boundary $(p \geq 1)$ remains an open question.

In the rest of this paper, we will be mainly concerned by the numerical solving of the system (2.15).

3. An iterative strategy. Our aim in this section is to propose a fixed point strategy for computing solutions of problem (2.15). The main idea consists to split this problem into two subproblems: a first order hyperbolic equation on $\lambda$ and a first order elliptic problem on $\boldsymbol{B}$. Let $(\boldsymbol{B}, \lambda(\boldsymbol{x}))$ be solution of (2.15) and set $\boldsymbol{\omega}=\operatorname{curl} \boldsymbol{B}$. Suppose that $\boldsymbol{B}$ is well known and is force-free. Then, another manner for computing $\boldsymbol{\omega}$ consists to set $\boldsymbol{\omega}=\lambda(\boldsymbol{x}) \boldsymbol{B}$ and then to calculate $\lambda(\boldsymbol{x})$ by solving the transport equation

$$
\boldsymbol{B} . \nabla \lambda(\boldsymbol{x})=0 \text { in } \Omega, \lambda=\alpha_{0}(\boldsymbol{x}) \text { on } \Sigma^{-} .
$$

Conversely, if $\boldsymbol{\omega}(\boldsymbol{x})$ is a given vector field satisfying $\operatorname{div} \boldsymbol{\omega}=0$ and $\langle\boldsymbol{\omega} \cdot \boldsymbol{n}, 1\rangle_{\Gamma_{i}}=0$, $0 \leq i \leq p$, then $\boldsymbol{B}$ can be obtained by solving the curl-div system

$$
\operatorname{curl} \boldsymbol{B}=\boldsymbol{\omega} \text { in } \Omega, \operatorname{div} \boldsymbol{B}=0 \text { in } \Omega, \boldsymbol{B} \cdot \boldsymbol{n}=g \text { on } \partial \Omega,
$$

(completed with suitable conditions when $\Omega$ is multiply-connected).

Combining these remarks leads to consider a first fixed point scheme: starting from an initial guess $\boldsymbol{B}_{0}$, the sequence $\left(\boldsymbol{B}_{n}, \lambda_{n}\right)$ is constructed as follows 
- For $n \geq 0, \lambda_{n}$ is solution to the transport equation

$$
\left\{\begin{aligned}
\boldsymbol{B}_{n} \cdot \nabla \lambda_{n} & =0 \text { in } \Omega \\
\lambda_{n} & =\alpha_{0} \text { on } \Sigma^{-}
\end{aligned}\right.
$$

- For $n \geq 0, \boldsymbol{B}_{n+1}$ is solution of the curl-div vectorial system

$$
\left\{\begin{aligned}
\operatorname{curl} \boldsymbol{B}_{n+1} & =\lambda_{n} \boldsymbol{B}_{n} & & \text { in } \Omega, \\
\operatorname{div} \boldsymbol{B}_{n+1} & =0 & & \text { in } \Omega, \\
\boldsymbol{B}_{n+1} \cdot \boldsymbol{n} & =g & & \text { on } \partial \Omega .
\end{aligned}\right.
$$

(completed with suitable conditions on cuts when $\Omega$ is not simply-connected). At each iteration of this process, one should therefore solve a usual vector potential problem (3.4) and a hyperbolic first order problem (3.3). The initial guess $\boldsymbol{B}_{0}$ can be taken as the curl-free and divergence free vector field associated to $g$, that is $\boldsymbol{B}_{0}$ is solution of (2.10).

Here, we consider a more general algorithm. This algorithm is based on a perturbation of the transport equation (3.3) by adding an artificial reaction term in the iterative process. More precisely, we consider the singularly perturbed algorithm: starting from $\boldsymbol{B}_{0}$, we construct a sequence $\left(\boldsymbol{B}_{n}, \lambda_{n}\right)$ as follows

- For $n \geq 0, \lambda_{n}$ is solution of the system

$$
\left\{\begin{aligned}
\boldsymbol{B}_{n} \cdot \nabla \lambda_{n}+\sigma_{n} \lambda_{n} & =f_{n-1} \text { in } \Omega \\
\lambda_{n} & =\alpha_{0} \text { on } \Sigma^{-}
\end{aligned}\right.
$$

where $f_{n-1}=\sigma_{n} \lambda_{n-1}$ if $n \geq 1$ and $f_{-1}=0$. Here $\left(\sigma_{n}\right)_{n \geq 0}$ is a sequence of parameters chosen such that, $\sigma_{n}>0$, for all $n$.

- For $n \geq 1, \boldsymbol{B}_{n}$ is solution of the second order vectorial problem

$$
\left\{\begin{array}{rlrl}
\operatorname{curl} \operatorname{curl} \boldsymbol{B}_{n} & =\operatorname{curl} \boldsymbol{j}_{n-1} & & \text { in } \Omega, \\
\operatorname{div} \boldsymbol{B}_{n} & =0 & & \text { in } \Omega, \\
\boldsymbol{B}_{n} \cdot \boldsymbol{n} & =g & & \text { on } \partial \Omega, \\
\left(\operatorname{curl} \boldsymbol{B}_{n}-\boldsymbol{j}_{n-1}\right) \times \boldsymbol{n} & =\mathbf{0} & & \text { on } \partial \Omega, \\
\int_{\Sigma_{i}}^{\boldsymbol{B}_{n} . \boldsymbol{n} d \sigma} & =a_{i}, \forall i \in\{1, \ldots, m\}, &
\end{array}\right.
$$

where $\boldsymbol{j}_{n-1}=\lambda_{n-1} \boldsymbol{B}_{n-1}$. The starting guess $\boldsymbol{B}_{0}$ is still chosen as the irrotational field associated to $g$ defined by the system (2.10).

This algorithm is more general than the first one since we shall prove in section 4.1 that (3.6) is equivalent to the vector potential problem

$$
\left\{\begin{array}{rlrl}
\operatorname{curl} \boldsymbol{B}_{n} & =\boldsymbol{j}_{n-1}^{*} & & \text { in } \Omega, \\
\operatorname{div} \boldsymbol{B}_{n} & =0 & & \text { in } \Omega, \\
\boldsymbol{B}_{n} \cdot \boldsymbol{n} & =g & & \text { on } \partial \Omega, \\
\int_{\Sigma_{i}} \boldsymbol{B}_{n} \cdot \boldsymbol{n} d \sigma & =a_{i}, \forall i \in\{1, \ldots, m\}, &
\end{array}\right.
$$

where $\boldsymbol{j}_{n-1}^{*}=\boldsymbol{j}_{n-1}+\nabla q_{n-1}$, with $\boldsymbol{j}_{n-1}=\lambda_{n-1} \boldsymbol{B}_{n-1}$. Here $q_{n-1}$ is chosen such that the right hand side is a curl. The first algorithm corresponds to the case $\sigma_{n}=0$ and $q_{n}=0$ for $n \geq 0$. 
On the other hand, setting $\boldsymbol{b}_{n}=\boldsymbol{B}_{n}-\boldsymbol{B}_{0}$, the system (3.6) is reduced to

$$
\left\{\begin{aligned}
\operatorname{curl} \operatorname{curl} \boldsymbol{b}_{n} & =\operatorname{curl} \boldsymbol{j}_{n-1} & & \text { in } \Omega, \\
\operatorname{div} \boldsymbol{b}_{n} & =0 & & \text { in } \Omega, \\
\boldsymbol{b}_{n} \cdot \boldsymbol{n} & =0 & & \text { on } \partial \Omega, \\
\left(\operatorname{curl} \boldsymbol{b}_{n}-\boldsymbol{j}_{n-1}\right) \times \boldsymbol{n} & =\mathbf{0} \text { on } \partial \Omega, & & \\
\int_{\Sigma_{i}} \boldsymbol{b}_{n} \cdot \boldsymbol{n} d \sigma & = & 0, \forall i \in\{1, \ldots, m\} . &
\end{aligned}\right.
$$

In summary, at each iteration of the algorithm one should solve two problems:

(a) A reaction-convection problem of the form: find $\lambda$ solution of

$$
\left\{\begin{aligned}
\operatorname{div}(\lambda \boldsymbol{B})+\sigma \lambda & =f \text { in } \Omega \\
\lambda & =h \text { on } \Sigma^{-} .
\end{aligned}\right.
$$

(b) A vector potential problem: find a vector field $\boldsymbol{b}$ such that

$$
\left\{\begin{aligned}
\operatorname{curl} \operatorname{curl} \boldsymbol{b} & =\operatorname{curl} \boldsymbol{j} \text { in } \Omega \\
\operatorname{div} \boldsymbol{b} & =0 \text { in } \Omega \\
\boldsymbol{b . n} & =0 \text { on } \partial \Omega \\
(\operatorname{curl} \boldsymbol{b}-\boldsymbol{j}) \times \boldsymbol{n} & =\mathbf{0} \text { on } \partial \Omega \\
\int_{\Sigma_{i}} \boldsymbol{b . n} d \sigma & =0, \text { for } i=1, \ldots, m
\end{aligned}\right.
$$

We shall prove that this problem is equivalent to the first-order mixed problem: find a pair $(\boldsymbol{b}, \epsilon)$ satisfying

$$
\left\{\begin{aligned}
\operatorname{curl} \boldsymbol{b}-\nabla \epsilon & =\boldsymbol{j} \text { in } \Omega \\
\operatorname{div} \boldsymbol{b} & =0 \text { in } \Omega \\
\boldsymbol{b} . \boldsymbol{n}= & 0 \text { on } \partial \Omega \\
\int_{\Sigma_{i}} \boldsymbol{b . n} d \sigma & =0, \text { for } i=1, \ldots, m \\
\epsilon & \text { is } \quad \text { constant on } \Gamma_{i}, \text { for } i=0, \ldots, p \\
\left\langle\frac{\partial \epsilon}{\partial n}, 1\right\rangle_{\Gamma_{i}} & =-\langle\boldsymbol{j} . \boldsymbol{n}, 1\rangle_{\Gamma_{i}}, \text { for } i=0, \ldots, p .
\end{aligned}\right.
$$

The system (3.9) is a first order hyperbolic system. A general approach to that kind of problems can be found in [10] or in [4] where the existence of a solution to (3.9) when $\sigma>0$ and $\operatorname{div} \boldsymbol{B}=0$ is proved.

The convergence of this iterative process is not an easy matter. We conjecture that it converges if $\left|\alpha_{0}\right|_{\infty}<\mu_{0}$. Nevertheless, in the case of linear Beltrami flows $(\lambda$ is fixed and known), Boulmezaoud and Amari [16] proved that this process is superconvergent: the distance between $\boldsymbol{B}^{(n)}$ and the solution $\boldsymbol{B}$ is exponentially decreasing with $n$. It is worth noting that the convergence could be lost if $\left|\alpha_{0}\right|_{\infty} \geq \mu_{0}$.

Notice that at convergence we get

$$
\left\{\begin{array}{rlrl}
\operatorname{curl}(\operatorname{curl} \boldsymbol{B}-\lambda \boldsymbol{B}) & =\mathbf{0} & & \text { in } \Omega, \\
\operatorname{div} \boldsymbol{B} & =0 & & \text { in } \Omega, \\
\boldsymbol{B} \cdot \boldsymbol{n} & =g & & \text { on } \partial \Omega, \\
(\operatorname{curl} \boldsymbol{B}-\lambda \boldsymbol{B}) \times \boldsymbol{n} & =\mathbf{0} & & \text { on } \partial \Omega, \\
\int_{\Sigma_{i}} \boldsymbol{B}_{n} . \boldsymbol{n} d \sigma & =a_{i}, \forall i \in\{1, \ldots, m\}, &
\end{array}\right.
$$


In other words, there exists a function $\epsilon$ belonging to the space

$$
G=\left\{\theta \in H^{1}(\Omega) \cap L_{0}^{2}(\Omega) \mid \Delta \theta=0 \text { in } \Omega, \theta \text { is constant on } \Gamma_{i}, \text { for } i=0, \ldots, p\right\} .
$$

such that

$$
\left\{\begin{array}{rlrl}
\operatorname{curl} \boldsymbol{B}-\lambda \boldsymbol{B} & =\nabla \epsilon & & \text { in } \Omega, \\
\operatorname{div} \boldsymbol{B} & =0 & & \text { in } \Omega, \\
\boldsymbol{B} \cdot \boldsymbol{n} & =g & & \text { on } \partial \Omega, \\
\int_{\Sigma_{i}} \boldsymbol{B}_{n} \cdot \boldsymbol{n} d \sigma & =a_{i}, \forall i \in\{1, \ldots, m\}, &
\end{array}\right.
$$

When $\partial \Omega$ is connected $(p=0)$, the space $G$ is reduced to $\{\mathbf{0}\}$. Thus $\epsilon=0$ and $\boldsymbol{B}$ is force-free.

When $p \geq 1$, one can prove easily the identities (see Lemma 4.5)

$$
\left\langle\frac{\partial \epsilon}{\partial n}, 1\right\rangle_{\Gamma_{i}}=-\langle\lambda \boldsymbol{B} . \boldsymbol{n}, 1\rangle_{\Gamma_{i}}=-\langle\lambda g, 1\rangle_{\Gamma_{i}}, i=0, \ldots, p .
$$

On the other hand, the space $G$ is finite dimensional and $\operatorname{dim} G=p$. More precisely, $G$ is spanned by the functions $\epsilon_{1}-\overline{\epsilon_{1}}, \ldots, \epsilon_{p}-\overline{\epsilon_{p}}$ where, for $0 \leq i \leq p, \epsilon_{i}$ is the unique function of $G$ satisfying $\epsilon_{i}=\delta_{i, j}$ on $\Gamma_{j}, 1 \leq j \leq p$. According to the identity,

$$
\int_{\Omega}|\nabla \epsilon|^{2}=\sum_{i=0}^{p} \epsilon_{\mid \Gamma_{i}}\left\langle\frac{\partial \epsilon}{\partial n}, 1\right\rangle_{\Gamma_{i}},
$$

and (3.14) one deduces that $\epsilon=0$ (and $\operatorname{curl} \boldsymbol{B}=\lambda \boldsymbol{B}$ ) if and only if

$$
\forall 0 \leq i \leq p,\langle\lambda g, 1\rangle_{\Gamma_{i}}=0 .
$$

This condition obviously valid when $\lambda$ is a non zero constant and $g$ satisfying (2.13).

In the other cases, namely when $p \geq 1$ and $\lambda$ is non-constant, it is not sure whether the function $\lambda$ obtained after convergence satisfies (3.15) or not. The only information we get is that $\langle\lambda g, 1\rangle_{\partial \Omega}=\sum_{i=0}^{p}\langle\lambda g, 1\rangle_{\Gamma_{i}}=0$. For example, if there exists $k \leq p$ such that $\Gamma_{k} \subset \Sigma^{-}$and if $\left\langle\lambda_{0} g, 1\right\rangle_{\Gamma_{k}} \neq 0$, then (3.15) is not fulfilled and $\boldsymbol{B}$ is not Beltrami. Roughly speaking, condition (3.15) seems to be satisfied if for each $i \leq p$, the fieldlines starting from $\Sigma^{-} \cap \Gamma_{i}$ return to $\Gamma_{i}$. The existence of fieldlines linking two different components of the boundary compromises the vanishing residual function $\epsilon$. In particular, it is not clear whether condition (2.13) is sufficient or not to get a Beltrami field at convergence.

4. The finite elements discretization. The calculation of a force free field by this algorithm needs to handle with three issues: (1) computation of the starting guess $\boldsymbol{B}_{0}(2)$ solving the hyperbolic problem (3.5) at each iteration (3) solving the elliptic problem (3.11) at each iteration. Our task line here is to go into details of the numerical methods we propose for solving these problems.

Throughout this paragraph, we assume that $\Omega$ is a bounded polyhedron in $\mathbb{R}^{3}$. We consider a family of triangulations $\left(\mathscr{T}_{h}\right)$ where each $\left(\mathscr{T}_{h}\right)$ is a set of tetrahedron $K \subset \Omega$ such that

(a) $\bar{\Omega}=\cup_{K \in \mathscr{T}_{h}} \bar{K}$.

(b) The intersection of two different tetrahedra is either empty or a corner or a face or an edge of both of them.

Here $h$ stands for the usual discretization parameter defined as the largest diameter of all the tetrahedra $K \in \mathscr{T}_{h}$. We assume in addition that all the tringulations $\left(\mathscr{T}_{h}\right)$ satisfies the usual regularity assumptions (see [24]). 
4.1. The discretization of the potential field problem. Our intention in this section is to focus on the approximation of the potential field $\boldsymbol{B}_{0}$, solution of problem (2.10). A first idea consists to rewrite $\boldsymbol{B}_{0}$ as a gradient $\boldsymbol{B}_{0}=\nabla \Phi$ (when $\Omega$ is simply connected). One is lead to solve the celebrated Laplace equation

$$
-\Delta \Phi=f \text { in } \Omega \frac{\partial \Phi}{\partial n}=g \text { on } \partial \Omega
$$

with $f=0$ in our case. In our context, several constraints must be taken into account. For example, we need an accurate approximation of $\Delta \Phi$ since it represents the divergence of $\boldsymbol{B}_{0}$. In particular, the normal traces of the discretized potential field $\boldsymbol{B}_{0 h}=\nabla \Phi_{h}$ must be continuous at the interfaces between any two adjoining elements. In summary, emphasis must be put not only on the approximation of $\Phi$, but also on the approximation of $\boldsymbol{B}_{0}=\nabla \Phi$. We also need to keep the boundary condition $\boldsymbol{B}_{0} . \boldsymbol{n}=g$ after discretisation: in other word, we would like to have

$$
\operatorname{div} \boldsymbol{B}_{0 h}=0 \text { in } \Omega \text { and } \boldsymbol{B}_{0 h} . \boldsymbol{n}=g_{h} \text { on } \partial \Omega
$$

where $g_{h}$ is the interpolated boundary function $g$. Lastly, one must keep in mind that the used method has to be efficient and practical, since we are dealing with a $3 \mathrm{D}$ problem.

For all these reasons, the used standard finite element approximation of problem (4.1) (called the primal formulation) is not adequate, unless that high order methods, with a huger number of degrees of freedom are used. At the contrary, mixed methods could be prefered precisely because of their emphasis on the approximation of both of $\Phi$ and its gradient.

Define

$$
W^{g}(\Omega)=\left\{\boldsymbol{w} \in H(\operatorname{div}, \Omega) ; \boldsymbol{w} \cdot \boldsymbol{n}=g \text { in } H^{-1 / 2}(\partial \Omega)\right\}
$$

Then,

$$
W^{0}(\Omega)=H_{0}(\operatorname{div}, \Omega) .
$$

We set $\boldsymbol{p}=\nabla \Phi$. Since the map

$$
\boldsymbol{v} \in H(\operatorname{div} ; \Omega) \rightarrow \boldsymbol{v} . \boldsymbol{n} \in H^{-1 / 2}(\partial \Omega)
$$

is onto, we can find $\boldsymbol{p}_{g} \in H(\operatorname{div} ; \Omega)$ such that $\boldsymbol{p}_{g} \cdot \boldsymbol{n}=g$ in $H^{-1 / 2}(\partial \Omega)$. We can write $\boldsymbol{p}$ in the form $\boldsymbol{p}=\boldsymbol{p}_{g}+\boldsymbol{p}_{0}$ with $\boldsymbol{p}_{0} \in W^{0}(\Omega)$. Problem (4.1) can be reformulated into the mixed form

$$
\left\{\begin{array}{l}
\text { Find } \boldsymbol{p}_{0} \in W^{0}, \text { and } \Phi \in L_{0}^{2}(\Omega) \text { such that } \\
\forall \boldsymbol{q} \in W^{0}, \quad \int_{\Omega} \boldsymbol{p}_{0} \cdot \boldsymbol{q}+\int_{\Omega} \Phi \operatorname{div} \boldsymbol{q}=-\int_{\Omega} \boldsymbol{p}_{g} \cdot \boldsymbol{q} \\
\forall v \in L_{0}^{2}(\Omega), \quad \int_{\Omega} v \operatorname{div} \boldsymbol{p}_{0}=-\int_{\Omega} f v-\int_{\Omega} v \operatorname{div} \boldsymbol{p}_{g} .
\end{array}\right.
$$

We have the following lemma

Lemma 4.1. Problem (4.2) has one and only one solution $\left(\boldsymbol{p}_{0}, \Phi\right) \in W^{0} \times L_{0}^{2}(\Omega)$ and $\boldsymbol{p}=\nabla \phi$. The proof can be found in [47] or in Appendix A

Now, we interest in the discretization of the problem (4.2). Consider $W_{h}^{0}$ and $L_{h}^{0}$ two finite-dimensional subspace of $W^{0}(\Omega)$ and $L_{0}^{2}(\Omega)$ respectively, and $R T_{k}$ the polynomial space defined for each integer $k \geq 0$ by

$$
R T_{k}=\mathbb{P}_{k}^{3} \oplus \boldsymbol{x} \mathbb{H}_{k} \text {. }
$$


Define

$$
W_{h}^{0}=\left\{\boldsymbol{q} \in W^{0}(\Omega) \mid \forall K \in \mathscr{T}_{h}, \boldsymbol{q}_{\mid K} \in R T_{k}(K)\right\}
$$

and

$$
L_{h}^{0}=\left\{v \in L_{0}^{2}(\Omega), \quad v_{\mid K} \in \mathbb{P}_{k}(K)\right\}
$$

The discrete form of (4.2) writes

$$
\left\{\begin{array}{l}
\text { Find }\left(\boldsymbol{p}_{0 h}, \Phi_{h}\right) \in W_{h}^{0} \times L_{h}^{0} \text { such that } \\
\forall \boldsymbol{q}_{h} \in W_{h}^{0}, \quad \int_{\Omega} \boldsymbol{p}_{0 h} \cdot \boldsymbol{q}_{h}+\int \Phi_{h} \operatorname{div} \boldsymbol{q}_{h}=-\int_{\Omega} \boldsymbol{p}_{g} \cdot \boldsymbol{q}_{h} \\
\forall v_{h} \in L_{h}^{0}, \quad \int_{\Omega} v_{h} \operatorname{div} \boldsymbol{p}_{0 h}=\int_{\Omega} f v_{h}-\int_{\Omega} v_{h} \operatorname{div} \boldsymbol{p}_{g} .
\end{array}\right.
$$

In [47], Roberts and Thomas prove that the discrete inf-sup condition is uniformly statisfied and that the problem (4.3) has one and only one solution. However, the matrix arising from (4.3) is not positive definite although it is invertible.

Here, we propose to use a mixed hybrid finite element method (see [47], [20]). The main idea of this method is to remove the stress on the inter-element continuity by introduicing a Lagrange multiplier. Denote by $\partial \mathscr{T}_{h}$ the set of all faces of the mesh, and consider the spaces

$$
\begin{gathered}
W_{h}{ }^{*}=\left\{\boldsymbol{q}_{h} \in L^{2}(\Omega)^{3} \mid \forall K \in \mathscr{T}_{h}, \boldsymbol{q}_{h \mid K} \in R T_{k}(K)\right\}, \\
\Lambda_{h}^{*}=\left\{v_{h} \in L^{2}\left(\partial \mathscr{T}_{h}\right), \quad \forall T \in \partial \mathscr{T}_{h}, v_{h \mid T} \in \mathbb{P}_{k}(T)\right\},
\end{gathered}
$$

where $k \geq 0$ in an integer. We note by $T^{\prime} \in K$ the four faces of a tetrahedron $K$. Consider the problem

$$
\left\{\begin{array}{l}
\text { Find }\left(\boldsymbol{p}_{h}^{*}, \Phi_{h}^{*}, \lambda_{h}^{*}\right) \in W_{h}^{*} \times L_{h}^{0} \times \Lambda_{h}^{*} \text { such that } \\
\forall K \in \mathscr{T}_{h}, \forall \boldsymbol{q}_{h} \in R T_{k}(K), \quad \int_{K} \boldsymbol{p}_{h}^{*} \boldsymbol{q}_{h} d K+\int_{K} \Phi_{h}^{*} \operatorname{div} \boldsymbol{q}_{h}-\sum_{T^{\prime} \subset \partial \mathscr{T}_{h}} \int_{T^{\prime}} \lambda_{h}^{*} \boldsymbol{q}_{h} \cdot \boldsymbol{n} d T^{\prime}=0, \\
\forall K \in \mathscr{T}_{h}, \forall v \in \mathbb{P}_{k}(K), \quad \int_{K} \operatorname{div} \boldsymbol{p}_{h}^{*} v d K=-\int_{K} f v d K, \\
\forall \mu_{h} \in \Lambda_{h}, \sum_{K \in \mathscr{T}_{h}} \sum_{T^{\prime} \subset \partial K} \int_{T^{\prime}} \mu_{h} \boldsymbol{p}_{h}^{*} \cdot \boldsymbol{n} d T^{\prime}=G_{K}\left(\mu_{h}\right),
\end{array}\right.
$$

where $G_{K}$ is defined for each $K \in \mathscr{T}_{h}$ by

$$
G_{k}\left(\mu_{h}\right)=\left\{\begin{array}{l}
0 \text { if } K \cap \partial \Omega=\emptyset \\
\sum_{T^{\prime} \in K \cap \partial \Omega} \int_{T^{\prime}} g \mu_{h} d T^{\prime} \text { otherwise. }
\end{array}\right.
$$

The inter-element stress is imposed by the last equation of (4.4), and we have the following lemma

LEMMA 4.2. The problem (4.4) has one and only one solution $\left(\boldsymbol{p}_{h}^{*}, \Phi_{h}^{*}, \lambda_{h}^{*}\right) \in$ $W_{h}^{*} \times L_{h}^{0} \times \Lambda_{h}^{*}$. Moreover, the solution $\left(\boldsymbol{p}_{h}^{*}, \Phi_{h}^{*}\right)$ of the problem (4.4) is the same as the solution $\left(\boldsymbol{p}_{h}=\boldsymbol{p}_{0 h}+\boldsymbol{p}_{g}, \Phi_{h}\right)$ of (4.3).

Proof. 
Choosing $f=0$ and $g=0$ in the square system (4.4), and taking tests function in the space $W_{h}^{0} \subset W_{h}^{*}$ lead to formulation (4.3). Since (4.3) has one and only one solution, we deduce that $\boldsymbol{p}_{h}=\mathbf{0}$ and $\Phi_{h}=0$, and, using the first equation of (4.4), we get $\lambda_{h}=0$. $\square$ From a practical point of view, we follow the ideas proposed in [11] to reformulate the problem into a reduced form. The authors prove that

$$
R T_{k}=\left\{\boldsymbol{p} \in\left(\mathbb{P}_{k}\right)^{3} ; \operatorname{div} \boldsymbol{p}=0\right\} \oplus \boldsymbol{x} \mathbb{P}_{k} .
$$

Consider the space

$$
R T_{k}^{0}=\left\{\boldsymbol{p} \in R T_{k}(K) ; \operatorname{div} \boldsymbol{p}=0\right\} .
$$

According to [44], we have

$$
R T_{k}^{0}=\left\{\boldsymbol{p} \in\left(\mathbb{P}_{k}\right)^{3}, \operatorname{div} \boldsymbol{p}=0\right\} .
$$

We consider now the particular case where $k=0$. Choosing the test functions in $R T_{0}^{0}(K)$, the system (4.4) writes

$$
\left\{\begin{array}{l}
\text { Find }\left(\boldsymbol{p}_{h}, \lambda_{h}\right) \in W_{h}^{*} \times \Lambda_{h}^{*} \text { uch that } \\
\forall \boldsymbol{q} \in \mathbb{R}^{3}, \quad \int_{K} \boldsymbol{p}_{h} \cdot \boldsymbol{q}_{h} d K-\sum_{T^{\prime} \subset K} \int_{T^{\prime}} \lambda_{h} \boldsymbol{q}_{h} \cdot \boldsymbol{n} d T^{\prime}=0 \\
\int_{K} \operatorname{div} \boldsymbol{p}_{h} d K=\int_{K} f d K \\
\forall \mu_{h} \in \Lambda_{h}, \sum_{K \in \mathscr{T}_{h}} \sum_{T^{\prime} \subset \partial K} \int_{T^{\prime}} \mu_{h} \boldsymbol{p}_{h} \cdot \boldsymbol{n} d T^{\prime}=G_{K}\left(\mu_{h}\right),
\end{array}\right.
$$

for all $K \in \mathscr{T}_{h}$.

Taking $\boldsymbol{p}_{h \mid K}=\alpha_{K} \boldsymbol{x}+\boldsymbol{\beta}_{K}$, and using the equality $\int_{K} \operatorname{div} \boldsymbol{p}=-\int_{K} f$, one gets

$$
\alpha_{K}=-\frac{1}{3|K|} \int_{K} f d K
$$

The first equation of (4.5) becomes

$$
\int_{K}\left(\alpha_{K} \boldsymbol{x}+\boldsymbol{\beta}_{K}\right) \boldsymbol{q} d K-\sum_{T^{\prime} \subset K} \int_{T^{\prime}} \lambda_{h} \boldsymbol{q} \cdot \boldsymbol{n} d T^{\prime}=0, \quad \forall \boldsymbol{q} \in R T_{0}^{0} .
$$

Denote by $\boldsymbol{q}_{K}, \boldsymbol{\lambda}_{K}$ and $\boldsymbol{\mu}_{K}$ the vector column corresponding to $\boldsymbol{q} \in R T_{0}^{0}(K)$, $\left\{\lambda_{h \mid T^{\prime}}\right\}_{T^{\prime} \subset \partial K}$, and $\left\{\mu_{h \mid T^{\prime}}\right\}_{T^{\prime} \subset \partial K}$ respectively. The equation (4.6) writes

$$
|K| \boldsymbol{q}_{K}^{T} \boldsymbol{\beta}_{K}-\boldsymbol{q}_{K}^{T} C_{K} \boldsymbol{\lambda}_{K}=\boldsymbol{q}_{K}^{T}\left\{f_{K}^{(1)}\right\} .
$$

Here $C_{K}$ denotes the matrix of size $3 \times 4$ defined by

$$
C_{K}=\left(\begin{array}{cccc}
n_{11}\left|T_{1}\right| & n_{21}\left|T_{2}\right| & n_{31}\left|T_{3}\right| & n_{41}\left|T_{4}\right| \\
n_{12}\left|T_{1}\right| & n_{22}\left|T_{2}\right| & n_{32}\left|T_{3}\right| & n_{42}\left|T_{4}\right| \\
n_{13}\left|T_{1}\right| & n_{23}\left|T_{2}\right| & n_{33}\left|T_{3}\right| & n_{43}\left|T_{4}\right|
\end{array}\right)
$$

where $n_{i j}$ is the $j^{\text {th }}$ component of the unit exterior normal to the $i^{\text {th }}$ face of the tetrahedron $K$ (where the four faces a $K$ are denoted by $\left\{T_{1}, T_{2}, T_{3}, T_{4}\right\}$ ). The RHS is given by

$$
\left\{f_{K}^{(1)}\right\}=\frac{1}{3|K|}\left(\int_{K} f d K\right)\left(\int_{K} \boldsymbol{x} d K\right)
$$


We deduce that

$$
|K| \boldsymbol{\beta}_{K}-C_{K} \boldsymbol{\lambda}_{K}=f_{K}^{(1)}
$$

The last equation of (4.5) writes

$$
\text { For any } \boldsymbol{\mu} \in \Lambda_{h}^{*}, \sum_{K \in \mathscr{T}_{h}} \boldsymbol{\beta}_{K}^{T} C_{K}^{T} \boldsymbol{\mu}_{K}=-\sum_{K \in \mathscr{T}_{h}} \boldsymbol{\mu}_{K}^{T}\left\{f_{K}^{(2)}\right\}+\sum_{K \in \mathscr{T}_{h}} \boldsymbol{\mu}_{K}^{T} G_{K}
$$

where

$$
\boldsymbol{\mu}_{K}^{T}\left\{f_{K}^{(2)}\right\}=\sum_{T^{\prime} \subset \partial K} \int_{T^{\prime}} \alpha_{K} \mu_{h} \boldsymbol{x} . \boldsymbol{n} d T^{\prime}
$$

and

$$
\boldsymbol{\mu}_{K}^{T} G_{K}=\left\{\begin{array}{l}
0 \text { if } K \cap \partial \Omega=\emptyset \\
\sum_{T^{\prime} \in K \cap \partial \Omega} \int_{T^{\prime}} g \mu_{h} d T^{\prime} \text { otherwise. }
\end{array}\right.
$$

Multiplying (4.7) by $\boldsymbol{\mu}_{K}^{T} C_{K}^{T}$, and summing on each element $K$ of $\mathscr{T}_{h}$ gives

$$
\forall \mu_{h} \in \Lambda_{h}, \quad \sum_{K \in \mathscr{T}_{h}} \frac{1}{|K|} C_{K}^{T} C_{K}\left\{\boldsymbol{\lambda}_{K}\right\}=\sum_{K \in \mathscr{T}_{h}} f_{K}
$$

where

$$
f_{K}=-\frac{1}{|K|} C_{K}^{T}\left\{f_{K}^{(1)}\right\}-\left\{f_{K}^{(2)}\right\}+\left\{G_{K}\right\} .
$$

In the end, problem (4.4) is reduced to the smaller system (4.8). The original problem (2.10) corresponds to the case $f=0$.

4.2. The discretization of the vector potential problem. Our purpose here is to discuss in details the finite elements discretization of the vector-potential problem (3.10). We shall only consider the case of a simply-connected geometry. We start by proving that problem (3.10) has a simple mixed formulation in terms of $\boldsymbol{b}$ and a fictitious unknown $\theta=0$. On the other hand, we prove that it equivalent to a first order system in which an auxiliary unknown function $q$ appears.

In the remaining, $M$ denotes the following space

$$
M=H^{1}(\Omega) \cap L_{0}^{2}(\Omega) .
$$

We consider the mixed problem: Find a pair $(\boldsymbol{b}, \theta)$ in $H(\mathbf{c u r l} ; \Omega) \times M$ such that

$$
(\mathscr{Q})\left\{\begin{array}{l}
\forall \boldsymbol{w} \in H(\operatorname{curl} ; \Omega), \int_{\Omega} \operatorname{curl} \boldsymbol{b} \cdot \operatorname{curl} \boldsymbol{w} d x+\int_{\Omega} \boldsymbol{w} \cdot \nabla \theta d x=\int_{\Omega} \boldsymbol{j} \cdot \operatorname{curl} \boldsymbol{w} d x \\
\forall \mu \in M, \int_{\Omega} \boldsymbol{b} \cdot \nabla \mu d x=0,
\end{array}\right.
$$

Lemma 4.3. Suppose that $\boldsymbol{j} \in L^{2}(\Omega)^{3}$. The problem $(\mathscr{Q})$ admits one and only one solution $(\boldsymbol{b}, \theta) \in H(\operatorname{curl} ; \Omega) \times M$. Moreover, $\theta=0$, $\operatorname{div} \boldsymbol{b}=0$ and

$$
\|\boldsymbol{b}\|_{H(\operatorname{curl} ; \Omega)} \lesssim\|\boldsymbol{j}\|_{L^{2}(\Omega)}
$$


Proof. We set

$$
\begin{aligned}
a(\boldsymbol{u}, \boldsymbol{v}) & =\int_{\Omega} \operatorname{curl} \boldsymbol{u} . \operatorname{curl} \boldsymbol{v} d x \text { for } \boldsymbol{u}, \boldsymbol{v} \in H(\operatorname{curl} ; \Omega), \\
c(\boldsymbol{v}, \theta) & =\int_{\Omega} \boldsymbol{v} . \nabla \theta d x \text { for } \boldsymbol{w} \in H(\operatorname{curl} ; \Omega) \text { and } \theta \in M .
\end{aligned}
$$

On one hand, notice that $a(.,$.$) is a positive semi-definite symmetric and continuous$ bilinear form on $H(\operatorname{curl} ; \Omega) \times H(\operatorname{curl} ; \Omega)$. Notice also that the bilinear form $c(.,$. is continuous on $H(\mathbf{c u r l} ; \Omega) \times M$. On the other hand, consider the space

$$
\begin{aligned}
V & =\{\boldsymbol{v} \in H(\operatorname{curl} ; \Omega) \mid c(\boldsymbol{v}, \theta)=0, \forall \theta \in M\} \\
& =\left\{\boldsymbol{v} \in H(\operatorname{curl} ; \Omega) \mid \int_{\Omega} \boldsymbol{v} \cdot \nabla \theta d x=0, \forall \theta \in M\right\} .
\end{aligned}
$$

According to Green' formula

$$
\forall \boldsymbol{v} \in H(\operatorname{div} ; \Omega), \forall \theta \in H^{1}(\Omega), \int_{\Omega} \theta \operatorname{div} \boldsymbol{v} d x+\int_{\Omega} \nabla \theta . \boldsymbol{v} d x=\langle\boldsymbol{v} . \boldsymbol{n}, \theta\rangle_{\partial \Omega},
$$

one can prove easily that

$$
V=\{\boldsymbol{v} \in U \mid \operatorname{div} \boldsymbol{v}=0\}
$$

Next, we have

$$
\begin{aligned}
\forall \boldsymbol{v} \in V, a(\boldsymbol{v}, \boldsymbol{v}) & =\|\operatorname{curl} \boldsymbol{v}\|_{0, \Omega}^{2} \\
& =|\boldsymbol{v}|_{U}^{2} \\
& \geq C\|\boldsymbol{v}\|_{H(\operatorname{curl} ; \Omega)}^{2} .
\end{aligned}
$$

Thus, $a(.,$.$) is V$-elliptic. Moreover, taking $\boldsymbol{v}=\nabla \mu$ gives

$$
\begin{aligned}
\forall \mu \in M, \sup _{\boldsymbol{v} \in H(\operatorname{curl} ; \Omega)} \frac{(\boldsymbol{v}, \nabla \mu)}{\|\boldsymbol{v}\|_{H(\operatorname{curl} ; \Omega)}} & \geq \frac{(\nabla \mu, \nabla \mu)}{\|\nabla \mu\|_{H(\mathbf{c u r l} ; \Omega)}} \\
& =\|\nabla \mu\|_{0, \Omega}^{2} \\
& =|\mu|_{1, \Omega}^{2} .
\end{aligned}
$$

Thus, according to the celebrated Babuska-Brezzi theorem (see [9] and [18]. See also $[28])$, the problem $(\mathscr{Q})$ has a single solution $(\boldsymbol{b}, \theta)$. Taking $\boldsymbol{w}=\nabla \theta$ as a test function implies that $\theta=0$.

Proposition 4.4. Let $\boldsymbol{j} \in L^{2}(\Omega)^{3}$ and $\boldsymbol{b} \in H(\mathbf{c u r l} ; \Omega)$. The following assertions are equivalent

(i) $(\boldsymbol{b}, 0)$ is solution of $(\mathscr{Q})$.

(ii) $\boldsymbol{b}$ is solution of (3.10).

(iii) There exists $\epsilon \in L_{0}^{2}(\Omega) \cap H^{1}(\Omega)$ such that the pair $(\boldsymbol{b}, \epsilon)$ is solution of (3.11). When these assertions hold, the function $\epsilon$ is the unique solution in $L_{0}^{2}(\Omega) \cap H^{1}(\Omega)$ of the problem

$$
\left\{\begin{aligned}
-\Delta \epsilon & =\operatorname{div} \boldsymbol{j} \text { in } \Omega, \\
\epsilon & \text { is constant on } \Gamma_{i}, \text { for } i=0, \ldots, p, \\
\langle(\nabla \epsilon+\boldsymbol{j}) \cdot \boldsymbol{n}, 1\rangle_{\Gamma_{i}} & =0, \text { for } i=0, \ldots, p .
\end{aligned}\right.
$$


(notice that necessarily $\nabla \epsilon+\boldsymbol{j} \in H(\operatorname{div} ; \Omega)$ and the last boundary condition is meaningful).

Proof. (of Proposition 4.4) We need the following lemma (see [28] Theorem 3.4 and [17] Lemma 5)

Lemma 4.5. Let $\boldsymbol{w} \in H(\operatorname{curl} ; \Omega)$. Then,

$$
\langle\operatorname{curl} \boldsymbol{w} \cdot \boldsymbol{n}, 1\rangle_{\Gamma_{i}}=0, \text { for } 0 \leq i \leq p .
$$

(notice that $\operatorname{curl} \boldsymbol{w} \in H(\operatorname{div}, \Omega)$ ). Conversely, if a vector field $\boldsymbol{v} \in L^{2}(\Omega)^{3}$ satisfies

$$
\operatorname{div} \boldsymbol{v}=\mathbf{0} \text { in } \Omega,\langle\boldsymbol{v} . \boldsymbol{n}, 1\rangle_{\Gamma_{i}}=0, i=0, \ldots, p .
$$

Then, there exists one and only one $\boldsymbol{w} \in L^{2}(\Omega)^{3}$ such that

$$
\operatorname{curl} \boldsymbol{w}=\boldsymbol{v}, \operatorname{div} \boldsymbol{w}=0, \boldsymbol{w} \cdot \boldsymbol{n}=0 \text { on } \partial \Omega .
$$

(the domain is supposed simply-connected). The equivalence (i) $\Longleftrightarrow$ (ii) in Proposition 4.4 is a simple exercice. Let us prove the equivalence of (i) and (iii).

(iii) $\Longrightarrow$ (i) Let $(\boldsymbol{b}, \epsilon)$ be solution of $(3.11)$ and $\boldsymbol{w}$ an arbitrary element of $H(\mathbf{c u r l}, \Omega)$. Then,

$$
\int_{\Omega} \nabla \epsilon . \operatorname{curl} \boldsymbol{w} d \boldsymbol{x}=\sum_{i=0}^{p}\langle\operatorname{curl} \boldsymbol{w} . \boldsymbol{n}, \epsilon\rangle_{\Gamma_{i}}=\sum_{i=0}^{p} \epsilon_{\mid \Gamma_{i}}\langle\operatorname{curl} \boldsymbol{w} . \boldsymbol{n}, 1\rangle_{\Gamma_{i}} .
$$

According to Lemma 4.5 we deduce that

$$
\int_{\Omega} \nabla \epsilon \cdot \operatorname{curl} \boldsymbol{w} d \boldsymbol{x}=0
$$

Multiplying the first equation of (3.11) by $\operatorname{curl} \boldsymbol{w}$ and using (4.11) gives the first identity of $(\mathscr{Q})$. The second identity of $(\mathscr{Q})$ is obtained after multiplying the equation $\operatorname{div} \boldsymbol{b}=0$ by an arbitrary $\mu \in M$.

(i) $\Longrightarrow$ (iii) Conversely, let $(\boldsymbol{b}, 0)$ be solution of $(\mathscr{Q})$ and consider the space

$$
\mathscr{V}=\left\{\boldsymbol{v} \in \mathscr{D}(\Omega)^{3} ; \operatorname{div} \boldsymbol{v}=0\right\}
$$

For each $\varphi \in \mathscr{V}$, there exists a vector potential $\boldsymbol{A}$ in $H^{1}(\Omega)^{3}$ (see [28]) such that $\boldsymbol{\varphi}=\operatorname{curl} \boldsymbol{A}$. Replacing $\boldsymbol{w}$ by $\boldsymbol{A}$ in (Q2) gives

$$
(\operatorname{curl} b-j, \varphi)=0, \forall \varphi \in \mathscr{V}
$$

Hence, there exists a unique function $\epsilon \in L_{0}^{2}(\Omega)$ such that $\nabla \epsilon=\operatorname{curl} \boldsymbol{b}-\boldsymbol{j}$. Moreover, $\epsilon$ is in $H^{1}(\Omega)$. The first part of $(\mathscr{Q})$ becomes

$$
\forall \boldsymbol{w} \in H(\operatorname{curl} ; \Omega), \quad(\nabla \epsilon, \operatorname{curl} \boldsymbol{w})=0 .
$$

According to Green's formula, we get

$$
\forall \boldsymbol{w} \in H(\operatorname{curl} ; \Omega), \sum_{i=0}^{p}\langle\operatorname{curl} \boldsymbol{w} \cdot \boldsymbol{n}, \epsilon\rangle_{\Gamma_{i}}=0 .
$$

For each $i \leq p$, define the constant

$$
\bar{q}_{i}=\frac{1}{\left|\Gamma_{i}\right|} \int_{\Gamma_{i}} \epsilon d \sigma
$$


where $\left|\Gamma_{i}\right|$ is the measure of $\Gamma_{i}$. According to Lemma 4.5 we deduce that

$$
\forall \boldsymbol{w} \in H(\operatorname{curl} ; \Omega), \sum_{i=0}^{p}\left\langle\operatorname{curl} \boldsymbol{w} . \boldsymbol{n}, \epsilon-\bar{q}_{i}\right\rangle_{\Gamma_{i}}=0 .
$$

Let $\boldsymbol{z}=\nabla \chi$, with $\chi$ solution to the Neumann problem

$$
\Delta \chi=0 \text { in } \Omega \text { and } \frac{\partial \chi}{\partial n}=\epsilon-\bar{q}_{i} \text { on } \partial \Omega .
$$

Since $\operatorname{div} \boldsymbol{z}=\mathbf{0}$ and $\langle\boldsymbol{z} . \boldsymbol{n}, 1\rangle_{\Gamma_{i}}=0$ for $0 \leq i \leq p$, there exists a vector function $\boldsymbol{w}_{0}$ in $H(\operatorname{curl} ; \Omega)$ such that $\operatorname{curl} \boldsymbol{w}_{0}=\boldsymbol{z}$. Replacing in (4.13) $\boldsymbol{w}$ by $\boldsymbol{w}_{0}$ gives

$$
\sum_{i=1}^{p} \int_{\Gamma_{i}}\left(\epsilon-\bar{q}_{i}\right)^{2} d \sigma=0 .
$$

In other words, $\epsilon=\bar{q}_{i}$ almost everywhere on $\Gamma_{i}$. It follows that $\epsilon$ is constant on each component $\Gamma_{i}, 0 \leq i \leq p$.

Identity $\nabla \epsilon=\operatorname{curl} \boldsymbol{b}-\boldsymbol{j}$ implies that

$$
-\Delta \epsilon=\operatorname{div} \boldsymbol{j} \in \Omega,\left\langle\frac{\partial \epsilon}{\partial n}, 1\right\rangle_{\Gamma_{i}}=-\langle\boldsymbol{j} . \boldsymbol{n}, 1\rangle_{\Gamma_{i}}, \text { for } 0 \leq i \leq p .
$$

We conclude that $\epsilon$ is solution of (4.10). It remains to prove that this solution is unique. Let $\epsilon^{\prime} \in L_{0}^{2}(\Omega)$ be another solution of (4.10) and set $\boldsymbol{v}=\boldsymbol{j}+\nabla \epsilon^{\prime}$. Then,

$$
\operatorname{div} \boldsymbol{v}=0,\langle\boldsymbol{v} . \boldsymbol{n}, 1\rangle_{\Gamma_{i}}=0 \text { for } i=0, \ldots, p .
$$

According to Lemma 4.5, there exists one and only one $\boldsymbol{b}^{\prime} \in L^{2}(\Omega)^{3}$ such that

$$
\operatorname{curl} \epsilon^{\prime}=\boldsymbol{v}, \operatorname{div} \epsilon^{\prime}=0, \epsilon^{\prime} \cdot \boldsymbol{n}=0 \text { on } \partial \Omega .
$$

The pair $\left(\boldsymbol{b}^{\prime}, \epsilon^{\prime}\right)$ is solution of (3.11). This implies that $\left(\boldsymbol{b}^{\prime}, 0\right)$ is solution of $(\mathscr{Q})$. Since $\boldsymbol{b}$ is the unique solution of $(\mathscr{Q})$, one deduces that $\boldsymbol{b}^{\prime}=\boldsymbol{b}$ and $\nabla \epsilon^{\prime}=\operatorname{curl} \boldsymbol{b}^{\prime}-\boldsymbol{j}=\operatorname{curl} \boldsymbol{b}-\boldsymbol{j}=\nabla \epsilon$. Thus, $\epsilon^{\prime}=\epsilon$.

A substantial advantage of formulation $(\mathscr{Q})$ in comparaison with the first order system (3.11) is that problem (Q2) does not involve the artificial function $\epsilon$. In other words, the computation of the function $\epsilon$, introduced here for clarification, is useless for the unrolling of the algorithm. Hence, we only deal with the approximation of problem $(\mathscr{Q})$ the statement of which suggests clearly the use of $H$ (curl ; $\Omega$ ) elements.

Let $X_{h}$ be a finite-dimensional subspace of $H(\operatorname{curl} ; \Omega), M_{h}$ a finite-dimensional subspace of $M$ and define the space

$$
V_{h}=\left\{\boldsymbol{w}_{h} \in X_{h},\left(\boldsymbol{w}_{h}, \nabla \mu_{h}\right)=0, \forall \mu_{h} \in M_{h}\right\} .
$$

Assume that

$$
\left(\mathscr{H}_{1}\right) \quad\left\{\nabla \mu_{h}, \mu_{h} \in M_{h}\right\} \subset X_{h} .
$$

Assume also that there exists a constant $C>0$, independent of $h$ such as :

$$
\left(\mathscr{H}_{2}\right) \quad\left\|\boldsymbol{w}_{h}\right\|_{0, \Omega} \leq C\left\|\operatorname{curl} \boldsymbol{w}_{h}\right\|_{0, \Omega}, \forall \boldsymbol{w}_{h} \in V_{h}
$$


Next, consider the problem

$$
\left(\mathscr{Q}_{h}\right)\left\{\begin{array}{l}
\text { Find }\left(\boldsymbol{u}_{h}, \theta_{h}\right) \in X_{h} \times M_{h} \text { such as } \\
\forall \boldsymbol{w}_{h} \in X_{h}, \int_{\Omega} \operatorname{curl} \boldsymbol{u}_{h} \cdot \operatorname{curl} \boldsymbol{w}_{h} d x+\int_{\Omega} \boldsymbol{w}_{h} \cdot \nabla \theta_{h} d x=\int_{\Omega} \boldsymbol{j} . \operatorname{curl} \boldsymbol{w}_{h} d x, \\
\forall \mu_{h} \in M_{h}, \int_{\Omega} \boldsymbol{u}_{h} \cdot \nabla \mu_{h}=0 .
\end{array}\right.
$$

The reader can refer to Amrouche and al. [6] for a proof of the following assertion.

Proposition 4.6. The problem $\left(\mathscr{Q}_{h}\right)$ has a single solution $\left(\boldsymbol{b}_{h}, \theta_{h}\right)$. Furthermore, $\theta_{h}=0$ and,

$$
\left\|\boldsymbol{b}-\boldsymbol{u}_{h}\right\|_{V} \leq C_{1} \inf _{\boldsymbol{w}_{h} \in X_{h}}\left\|\boldsymbol{b}-\boldsymbol{w}_{h}\right\|_{V}
$$

where the constant $C_{1}$ does not depend on $h$. A simple manner for constructing the finite-dimensional spaces $X_{h}$ and $M_{h}$ satisfying the assumptions $\left(\mathscr{H}_{1}\right)$ and $\left(\mathscr{H}_{2}\right)$ is the use of $H$ (curl) conforming elements developed by Nédelec [44] (see, e. g., [28]). More precisely, let $\ell \geq 1$ be an integer and consider the subspace of $\left(\mathbb{P}_{\ell}\right)^{3}$

$$
\begin{aligned}
& \mathscr{S}_{\ell}=\left\{p \in \mathbb{H}_{\ell}^{3}, \boldsymbol{p}(\boldsymbol{x}) \cdot \boldsymbol{x} \equiv 0, \boldsymbol{x}=\left(x_{1}, x_{2}, x_{3}\right)\right\} \\
& \mathscr{R}_{\ell}=\mathbb{P}_{\ell-1} \oplus \mathscr{S}_{\ell}
\end{aligned}
$$

Then, the spaces $X_{h}$ and $M_{h}$ can be defined as

$$
\begin{aligned}
& X_{h}=\left\{\boldsymbol{w}_{h} \in H(\mathbf{c u r l} ; \Omega), \boldsymbol{w}_{h \mid K} \in \mathscr{R}_{\ell}, \forall K \in \mathscr{T}_{h}\right\} \\
& M_{h}=\left\{\mu_{h} \in H^{1}(\Omega), \mu_{h \mid K} \in \mathbb{P}_{\ell}, \forall K \in \mathscr{T}_{h}, \text { and } \int_{\Omega} \mu_{h}=0\right\} .
\end{aligned}
$$

In that case, the following estimate on the upper bound of the interpolation error holds

$$
\inf _{\boldsymbol{w}_{h} \in W_{h}}\left\|\boldsymbol{v}-\boldsymbol{w}_{h}\right\|_{H(\mathbf{c u r l} ; \Omega)} \leq C h^{\ell}\left\{|\boldsymbol{v}|_{\ell, \Omega}+|\boldsymbol{v}|_{\ell+1, \Omega}\right\}, \text { for all } \boldsymbol{v} \in H^{\ell+1}(\Omega)
$$

Combining the latter with (4.15) yields an estimate of the error between $\boldsymbol{b}$ and $\boldsymbol{u}_{h}$

$$
\left\|\boldsymbol{b}-\boldsymbol{u}_{h}\right\|_{V} \leq C h^{\ell}\left\{|\boldsymbol{b}|_{\ell, \Omega}+|\boldsymbol{b}|_{\ell+1, \Omega}\right\}
$$

which is valid if $\boldsymbol{b} \in H^{\ell+1}(\Omega)$.

An important feature of the discrete system $\left(\mathscr{Q}_{h}\right)$ is that only the discrete vector field $\boldsymbol{u}_{h}$ is really unknown. Actually, the discrete function $\theta_{h}$ is a priori known since $\theta_{h}=0$. As we will see hereafter, this property can be exploited from a practical viewpoint; we will prove that the linear system obtained from $\left(\mathscr{Q}_{h}\right)$ can be reduced to a smaller positive definite symmetric linear system in which $\theta_{h}$ is eliminated.

Let $N_{1}=\operatorname{dim} X_{h}$ and $N_{2}=\operatorname{dim} M_{h}$. Consider a basis $\left(\boldsymbol{\omega}_{i}\right)_{1 \leq i \leq N_{1}}$ of $X_{h}$ and a basis $\left(\chi_{i}\right)_{1 \leq i \leq N_{2}}$ of $M_{h}$. Let $\left(\boldsymbol{b}_{h}, \theta_{h}\right)$ be solution of the problem $\left(\mathscr{Q}_{h}\right)$. Setting $\boldsymbol{b}_{h}(\boldsymbol{x})=\sum_{i=1}^{N_{1}} \alpha_{i} \boldsymbol{\omega}_{i}(\boldsymbol{x})$ and $\theta_{h}=\sum_{i=1}^{N_{2}} \beta_{i} \chi_{i}(\boldsymbol{x})$, one can rewrite $\left(\mathscr{Q}_{h}\right)$ into the form

$$
\left(\begin{array}{cc}
A & B^{T} \\
B & 0
\end{array}\right)\left(\begin{array}{l}
X \\
Y
\end{array}\right)=\left(\begin{array}{c}
C \\
0
\end{array}\right)
$$


where $A=\left(a_{i j}\right)_{1 \leq i, j \leq N_{1}}$ and $B=\left(b_{i j}\right)_{1 \leq i \leq N_{2}, 1 \leq j \leq N_{1}}$ are the matrices defined as

$$
\begin{aligned}
a_{i j} & =\int_{\Omega} \operatorname{curl} \boldsymbol{\omega}_{i} \cdot \operatorname{curl} \boldsymbol{\omega}_{j} d x, \quad 1 \leq i, j \leq N_{1}, \\
b_{i j} & =\int_{\Omega}^{\boldsymbol{\omega}_{i} . \nabla \chi_{j} d x, \quad 1 \leq i \leq N_{1}, 1 \leq j \leq N_{2},}
\end{aligned}
$$

$C=\left(c_{1}, \ldots, c_{N_{1}}\right)^{T}$ is the vector whose components are

$$
c_{i}=\int_{\Omega} \boldsymbol{j} \cdot \operatorname{curl} \boldsymbol{\omega}_{i} d x, \quad 1 \leq i \leq N_{1}
$$

and $X=\left(\alpha_{1}, \ldots, \alpha_{N_{1}}\right)^{T}$ and $Y=\left(\beta_{1}, \ldots, \beta_{N_{2}}\right)^{T}$ are the unknowns.

LEMMA 4.7. Let $\Lambda$ be a symmetric positive definite matrix of size $N_{2} \times N_{2}$. Then, the pair $(X, Y)$ is solution of the system (4.17) if and only if $Y=0$ and $X$ is solution of the symmetric positive definite system

$$
\left(A+B^{T} \Lambda B\right) X=C .
$$

It is worth nothing that the matrix $A$ is symmetric positive semi-definite, but $A$ is not definite neither invertible.

Proof. The matrices $A$ and $B^{T} \Lambda B$ are clearly positive semi-definite and so is the matrix $A+B^{T} \Lambda B$.

Let us prove that $A+B^{T} \Lambda B$ is invertible. Let $Z \in \mathbb{R}^{N_{1}}$ such that $\left(A+B^{T} \Lambda B\right) Z=0$. Then, necessarily $Z^{T} A Z+(B Z)^{T} \Lambda(B Z)=0$. Since both the terms in the last identity are positive, we deduce that $Z^{T} A Z=0$ and $(B Z)^{T} \Lambda(B Z)=0$. Thus $B Z=0$ since $\Lambda$ is positive definite, and necessarily $A Z=0$. Namely, $(Z, 0)$ is solution of (4.17) with $C=0$. Thus $Z=0$.

Now, observe that if $(X, Y)$ is the unique solution of (4.17), then $Y=0$ thanks to Proposition 4.6 and therefore $A X=C$ and $B X=0$. Hence, $X$ is also the unique solution to (4.18).

The approach of replacing (4.17) by (4.18) can be viewed as an augmented Lagrangian method. In our context, the main advantage of this approach is that number of unknowns is reduced. The choice of the matrix so as to minimize computational costs is beyond the scope of this paper. The interested reader can consult $[30,31]$ and references therein. In the numerical tests given below, $\Lambda$ is of the form $\rho I$, with $\rho$ properly chosen. Roughly speaking, the larger the coefficient $\rho$ the faster is the inversion and the larger the condition number of the matrix $A+\rho B^{T} B$. In other terms, $\rho$ must be chosen in intermediate range such that both the spectral properties and the conditioning of the matrix $A+\rho B^{T} B$ are satisfactory.

REMARK 4.1. It is possible to break up the composition of the matrices $A, B$ and $C$. Let $L_{h}$ be a finite dimensional subspace of $L^{2}(\Omega)^{3}$ such that $X_{h} \subset L_{h}$ and $\operatorname{curl} \boldsymbol{w}_{h} \in L_{h}$ for all $\boldsymbol{w}_{h} \in X_{h}$. Let $\left(\boldsymbol{z}_{i}\right)_{1 \leq i \leq \ell_{h}}$ be a basis of $L_{h}$ and consider the matrix $K=\left(k_{i, j}\right)_{1 \leq i, j \leq \ell_{h}}$ where $k_{i j}=\int_{\Omega} \boldsymbol{z}_{i} . \boldsymbol{z}_{j} d x$. Let $R$ and $G$ be the matrices of the operators curl : $X_{h} \longrightarrow L_{h}$ and $\nabla: M_{h} \longrightarrow X_{h}$ respectively. We have $R G=0$, thanks to assumption $\left(\mathscr{H}_{1}\right)$. Let $I_{*}$ be the matrix of the injection $X_{h} \hookrightarrow L_{h}$. One can easily prove that $A=R^{T} K R, B=G^{T} I_{*}^{T} K I_{*}, C=R^{T} K J$ where $J$ is the coordinate vector of $\boldsymbol{j}_{h} \in L_{h}$. Notice that $G^{T} A=0, A G=0$ and $G^{T} C=0$.

REMARK 4.2. Notice that $\operatorname{div} \boldsymbol{u}_{h} \neq 0$ in general since we use an $H(\mathbf{c u r l} ; \Omega)$ approximation. However, it is possible to construct a divergence-free approximation 
of $\boldsymbol{b}$ by projecting $\boldsymbol{b}_{h}$ on the space $H_{00}(\operatorname{div} ; \Omega)=\left\{\boldsymbol{v} \in L^{2}(\Omega)^{3} ; \operatorname{div} \boldsymbol{v}=0,\langle\boldsymbol{v} . \boldsymbol{n}, 1\rangle_{\Gamma_{i}}=\right.$ $0 \quad i=0, \ldots, p\}$. This projection can be constructed as follows; let $T$ be the operator which associates to each function $\boldsymbol{j} \in L^{2}(\Omega)^{3}$ the function $\boldsymbol{b} \in H(\mathbf{c u r l} ; \Omega)$ solution of $(\mathscr{Q})$. Consider also the operator

$$
\begin{aligned}
\Pi: L^{2}(\Omega)^{3} & \longrightarrow H_{00}(\operatorname{div} ; \Omega), \\
\boldsymbol{j} & \mapsto \operatorname{curl}(T \boldsymbol{j}) .
\end{aligned}
$$

Notice that $\Pi \boldsymbol{j}=\boldsymbol{j}$ if $\boldsymbol{j} \in H_{00}(\operatorname{div} ; \Omega)$, thanks to Proposition 4.4. Moreover, for all $\boldsymbol{j} \in L^{2}(\Omega)^{3}$ and all $\boldsymbol{v} \in H_{00}(\operatorname{div} ; \Omega)$ one has

$$
\begin{aligned}
& \int_{\Omega} \Pi \boldsymbol{j} \cdot \boldsymbol{v} d x=\int_{\Omega} \operatorname{curl}(T \boldsymbol{j}) \cdot \operatorname{curl}(T \boldsymbol{v}) d x \\
& =\int_{\Omega}^{\Omega} \boldsymbol{j} \cdot \operatorname{curl}(T \boldsymbol{v}) d x=\int_{\Omega} \boldsymbol{j} \cdot \boldsymbol{v} d x .
\end{aligned}
$$

Thus,

$$
\int_{\Omega}(\Pi \boldsymbol{j}-\boldsymbol{j}) . \boldsymbol{v} d x=0 \text { for all } \boldsymbol{v} \in H_{00}(\operatorname{div} ; \Omega)
$$

It follows that $\Pi$ is the projection operator from $L^{2}(\Omega)^{3}$ into $H_{00}(\operatorname{div} ; \Omega)$. Moreover, according to Proposition 4.4, there exists a function $q \in H^{1}(\Omega)$ such that $\Pi j=j+\nabla q$.

An approximation of the projector $\Pi$ can be deduced easily from the discretization of the operator $T$ exposed above. From a practical viewpoint the matrix of the projection $\Pi_{h}$ which approximates $\Pi$ is given by

$$
P=R\left(A+B^{T} \Lambda B\right)^{-1} R^{T} K .
$$

Here $R$ and $K$ are the matrices introduced in Remark 4.1, while $\Lambda$ is an arbitrary symmetric positive definite matrix. It is worth noting that $P$ does not depend on $\Lambda$, thanks to Lemma 4.7.

4.3. A streamline diffusion method for the hyperbolic problem on $\lambda$. Our task in this paragraph is to approximate the first order hyperbolic system

$$
\left\{\begin{aligned}
\boldsymbol{B} \cdot \nabla \lambda+\sigma \lambda & =f \text { in } \Omega \\
\lambda & =\alpha_{0} \text { on } \Sigma^{-} .
\end{aligned}\right.
$$

When $\sigma=0$, equation (4.19) means that the stream derivative of $\lambda$ vanishes, and $\lambda$ is constant along the fieldlines of $\boldsymbol{B}$. Such an equation could be solved by computing those filed lines. Namely, suppose that $\boldsymbol{B}$ is sufficiently smooth, and let $\boldsymbol{x}$ an arbitrary point in $\Omega$. Define the fieldline $(\boldsymbol{X}(s, \boldsymbol{x}))_{s \geq 0}$ by

$$
\frac{d \boldsymbol{X}(s, \boldsymbol{x})}{d s}=-\boldsymbol{B}(\boldsymbol{X}(s, \boldsymbol{x})), \boldsymbol{X}(0, \boldsymbol{x})=\boldsymbol{x}
$$

The minus sign before $\boldsymbol{B}(\boldsymbol{X}(s, \boldsymbol{x}))$ means that we are going back the fieldline toward the inflow boundary. Let $s_{0}(\boldsymbol{x})$ be the maximum time of existence of $\boldsymbol{X}(s, \boldsymbol{x})$ in $\bar{\Omega}$. If $s_{0}(\boldsymbol{x})$ is finite, i. e. if the fieldline $(\boldsymbol{X}(s, \boldsymbol{x}))_{s \geq 0}$ is not confined in $\Omega$ but encounters the boundary, then necessarly $\boldsymbol{X}\left(s_{0}(\boldsymbol{x}), \boldsymbol{x}\right)$ belongs to $\Sigma^{-}$and one can write

$$
\lambda(\boldsymbol{x})=\alpha_{0}\left(\boldsymbol{X}\left(s_{0}(\boldsymbol{x}), \boldsymbol{x}\right)\right) .
$$


In other words, $\lambda(\boldsymbol{x})$ is computed by going back the fieldlines of $\boldsymbol{B}$ originating from $\boldsymbol{x}$ until the boundary. In $[2,5]$, Amari and al. used this method for solving the problem on $\lambda(\boldsymbol{x})$ and a finite difference method for solving (3.1). Since the fact that the obtained numerical results are quite accurate, this algorithm has drawbacks. The first drawback is the high cost of computation which is mainly due to the integration of the fieldlines at all the nodes of the mesh and at each iteration of the algorithm. Secondly, the method rests on the assumption that the vector field $\boldsymbol{B}$ is sufficiently smooth (unless the concept of fieldlines is meaningless) and that all its fields lines encounter the boundary; namely $s_{0}(\boldsymbol{x})<+\infty$ for all $\boldsymbol{x} \in \bar{\Omega}$. From a theorical viewpoint, the last assumption is not always valid since lines of force may have be never ending, or close on themselves or have a finite length and point to a neutral point where $\boldsymbol{B}=\mathbf{0}$.

REMARK 4.3. If a vector field $\boldsymbol{B}$ satisfies a condition of the form

$$
\boldsymbol{B} . \boldsymbol{k} \geq \beta>0 \text { for each } \boldsymbol{x} \in \Omega,
$$

where $\boldsymbol{k}$ is constant vector and $\beta$ is a real, then necessarily almost its fieldlines encounter the boundary. Indeed, in that case, one can prove that the set $\left\{\boldsymbol{x} \in \Omega ; s_{0}(\boldsymbol{x})=\right.$ $+\infty\}$ is a null set (see, e. g., [8]).

It is well known that the direct application of a Galerkin finite elements method to the singularly perturbed problem (4.19) may lead to the appearance of spurious oscillations and instabilities. In the two last decades, several methods were proposed to remove this drawback (especially when a diffusion term $-\epsilon \Delta \lambda$ is added). Most of the proposed methods concern the $2 \mathrm{D}$ case and are based on adding a stabilization term to the discrete problem. Among these methods, one can recall the SUPG method (Streamline Upwind Galerkin Stabilization), called also the streamline diffusion method (see Brookes and Hughes [21]), the discontinuous Galerkin method (see Lesaint [39]) and the finite elements methods enriched with so-called bubble functions (see, e. g., Brezzi and al. [19]).

Here we shall use the method of streamline diffusion due to Brookes and Hughes [21] (see also, e. g., Johnson et al. [35]) which is based on a modification of the usual Galerkin. The discrete problem we consider is

$$
\left(\mathscr{P}_{h}\right)\left\{\begin{array}{l}
\text { Find } \lambda_{h} \in W_{h} \text { such that } \\
a_{h}\left(\lambda_{h}, w_{h}\right)=\ell_{h}\left(w_{h}\right), \forall w_{h} \in W_{h},
\end{array}\right.
$$

where

$$
\begin{aligned}
a_{h}\left(u_{h}, w_{h}\right) & =\int_{\Omega}\left(\boldsymbol{B} \cdot \nabla u_{h}+\sigma u_{h}\right) \cdot\left(w_{h}+\delta_{h} \boldsymbol{B} \cdot \nabla w_{h}\right) d x-\int_{\Sigma^{-}} u_{h} w_{h}(\boldsymbol{B} \cdot \boldsymbol{n}) d x \\
\ell_{h}\left(w_{h}\right) & =\int_{\Omega} f(\boldsymbol{x})\left(\delta_{h} \boldsymbol{B} \cdot \nabla w_{h}+w_{h}\right)-\int_{\Sigma^{-}} \alpha_{0} w_{h}(\boldsymbol{B} \cdot \boldsymbol{n}) d x .
\end{aligned}
$$

Here $W_{h}$ stands for the finite elements space

$$
W_{h}=\left\{v_{h} \in H^{1}(\Omega) ; v_{\mid K} \in \mathbb{P}_{k}(K), \forall K \in \mathscr{T}_{h}\right\},
$$

where for each $K \in \mathscr{T}_{h}, \mathbb{P}_{k}(K)$ denotes the space of polynomials of degree less or equal $k$. 
After few calculation, one can prove that

$$
\begin{aligned}
a_{h}\left(u_{h}, w_{h}\right)= & \delta_{h} \int_{\Omega}\left(\boldsymbol{B} \cdot \nabla u_{h}\right)\left(\boldsymbol{B} \cdot \nabla w_{h}\right) d x+\sigma \int_{\Omega} u_{h} w_{h} d x \\
& +\frac{1-\sigma \delta_{h}}{2} \int_{\Omega}\left[w_{h} \boldsymbol{B} \cdot \nabla u_{h}-u_{h} \boldsymbol{B} \cdot \nabla w_{h}\right] d x \\
& +\frac{1+\sigma \delta_{h}}{2} \int_{\partial \Omega \backslash \Sigma^{-}} u_{h} w_{h}(\boldsymbol{B} \cdot \boldsymbol{n})-\frac{1-\sigma \delta_{h}}{2} \int_{\Sigma^{-}} u_{h} w_{h}(\boldsymbol{B} \cdot \boldsymbol{n}) d x .
\end{aligned}
$$

We have clearly

$$
a_{h}\left(w_{h}, w_{h}\right) \geq \delta_{h}\left\|\boldsymbol{B} . \nabla w_{h}\right\|_{L^{2}(\Omega)}^{2}+\sigma\left\|w_{h}\right\|_{L^{2}(\Omega)}^{2}+\frac{\left(1-\delta_{h} \sigma\right)}{2}\left\|w_{h}\right\|_{L_{*}^{2}(\partial \Omega)}^{2},
$$

where

$$
\left\|w_{h}\right\|_{L_{*}^{2}(\partial \Omega)}^{2}=\int_{\partial \Omega}|\boldsymbol{B} \cdot \boldsymbol{n}| w_{h}^{2} d x .
$$

Hence,

$$
a_{h}\left(w_{h}, w_{h}\right) \geq \frac{1-\sigma \delta_{h}}{2}\left\|\left|w_{h} \|\right|_{\Omega}^{2},\right.
$$

if $\sigma \delta_{h}<1$. Here

$$
\left\|\left|w_{h}\left\|\left.\right|_{\Omega} ^{2}=\delta_{h}\right\| \boldsymbol{B} \cdot \nabla w_{h}\left\|_{L^{2}(\Omega)}^{2}+\sigma\right\| w_{h}\left\|_{L^{2}(\Omega)}^{2}+\right\| w_{h} \|_{L_{*}^{2}(\partial \Omega)}^{2} .\right.\right.
$$

The reader can refer to Appendix B for the proof of the following lemma:

LEMMA 4.8. Assume that if $\delta_{h} \sigma<1$. Then, the problem $\left(\mathscr{P}_{h}\right)$ has a single solution $\lambda_{h} \in V_{h}$. Moreover, if $\delta_{h}=$ ch for some constant c, $\boldsymbol{B} \in L^{\infty}(\Omega)^{3} \cap H(\operatorname{div} ; \Omega)$ and $\lambda \in H^{\ell+1}(\Omega)$ for some $\ell \geq 1$, then

$$
\left(1-\delta_{h} \sigma\right)\left\|\left|\lambda-\lambda_{h}\left\|\mid \leq C h^{\ell+1 / 2}\right\| \lambda \|_{H^{\ell+1}(\Omega)} .\right.\right.
$$

5. Computational results.. Our purpose in this section is to display some three-dimensional computational results, obtained with a three-dimensional code TETRAFFF containing a force-free fields solver in an arbitrary simply-connected domain. This solver uses the iterative algorithm of Section 3 and the finite elements methods exposed in Sections 4.2 and Section 4.3 respectively. Nédelec' edge elements of degree $\ell=1$ are used for solving the div-curl problem on $\boldsymbol{B}$. The hyperbolic problem on $\lambda$ is solved by means of $P^{1}$ conforming elements. The tests we made are divided into five categories.

The objective of the first experiences is to check seperately the convergence of the finite elements methods used for solving the vector-potential problem on $\boldsymbol{B}$ and the hyperbolic problem on $\lambda$.

The second family of tests concerns the convergence of the iterative fixed-point algorithm.

In the third tests, the emphasis is put on some particular properties of Beltrami flows. More precisely, we investigate the behavior of the the energy and the relative helicity of a Beltrami flow when it moves away from the irrotational one.

In the fourth tests, we propose to compute force-free fields in a cylindrical geometries, 
and to compare the non-linear solution with linear and irrotationnal solutions computed with the same boundary data.

In the last tests, we compare results obtained with our 3D code with the one obtained with the 2D axisymmetric code for toroidal magnetohydrodynamic equilibria (CHEASE) (see [43]) in toroidal geometry.

5.1. Preliminary experiences on convergence of the finite element methods. Here we test separately the convergence of the three problems involved in our algorithm : the mixed hybrid finite element method for solving the potential field problem, the $H$ (curl ) elements method used to solve the div-curl problem solutions (3.10) and the streamline diffusion method used to approximate the hyperbolic problem (4.19).

Preliminary test 1: Computation of the potential field. We assume here that $\Omega$ is a box $[-1,2] \times[-1,2] \times[0,1]$ and we consider the potential field created by a dipole situated under the plan $\{z=0\}$ at $z=-z_{0}$. The following magnetic field $\boldsymbol{B}_{0}$, introduced by Jackson (see [34]), will be used as an exact solution

$$
\begin{aligned}
& B_{0, x}^{e x}=3 b_{0} \frac{x_{1} z_{1}}{R^{5}} \\
& B_{0, y}^{e x}=3 b_{0} \frac{y_{1} z_{1}}{R^{5}} \\
& B_{0, z}^{e x}=b_{0} \frac{3 z_{1}^{2}-R^{2}}{R^{5}}
\end{aligned}
$$

where $x_{1}=x-\frac{a}{2}, y_{1}=y-\frac{b}{2}, z_{1}=z+z_{0}$ and $R=\sqrt{x_{1}^{2}+y_{1}^{2}+z_{1}^{2}}$. We choose $a=$ $b=5$ and $z_{0}=1$. We compute numerically the field $\boldsymbol{B}_{0}^{h}$ using $\boldsymbol{B}_{0}^{e x} \cdot \boldsymbol{n}$ as a boundary condition on $\partial \Omega$. In Table 5.1, we display the $L^{2}$ relative errors between the exact and the numerical solution. The errors decrease as $h^{0.96}$ and the convergence is linear.

\begin{tabular}{ccc}
\hline$h$ & DOF (faces) & $\left\|\boldsymbol{B}_{0}^{h}-\boldsymbol{B}_{0}^{e x}\right\|_{0, \Omega}$ \\
\cline { 3 - 3 } & & $\boldsymbol{B}_{0}^{e x} \|_{0, \Omega}$ \\
\hline 0.1563 & 16500 & 0.0871 \\
0.1172 & 390400 & 0.0671 \\
0.0938 & 760000 & 0.0543 \\
0.0781 & 1310400 & 0.0457 \\
\hline \multicolumn{3}{c}{ TABLE 5.1 }
\end{tabular}

$L^{2}$ relative error between the exact potential field and the numerical one obtained with the mixed hybrid finite element method.

In figure Fig.5.1, we display on the same figure some fieldlines of the magnetic field for the exact and a numerical solution obtained for $h=0.0866025$. The two solutions are in good agreement.

Preliminary test 2: the discretized curl-div system.. In all this second experience, $\Omega=B_{2}-\bar{B}_{1}$, where $B_{2}$ (resp. $B_{1}$ ) is the $3 \mathrm{D}$ sphere of radius 2 (resp. 1) and center $(0,0,0)$. In Table 5.2 , we display $L^{2}$ and $H$ (curl ) errors between a known exact solution $\boldsymbol{b}$ of (3.10) and the approximated one. The exact solution $(\boldsymbol{b}, q)$ of (3.10) is given by

$$
\boldsymbol{b}=\frac{\psi^{\prime}(r)}{r} \boldsymbol{x} \wedge(\boldsymbol{x} \wedge \boldsymbol{a})-2 \psi(r) \boldsymbol{a}, q=0,
$$




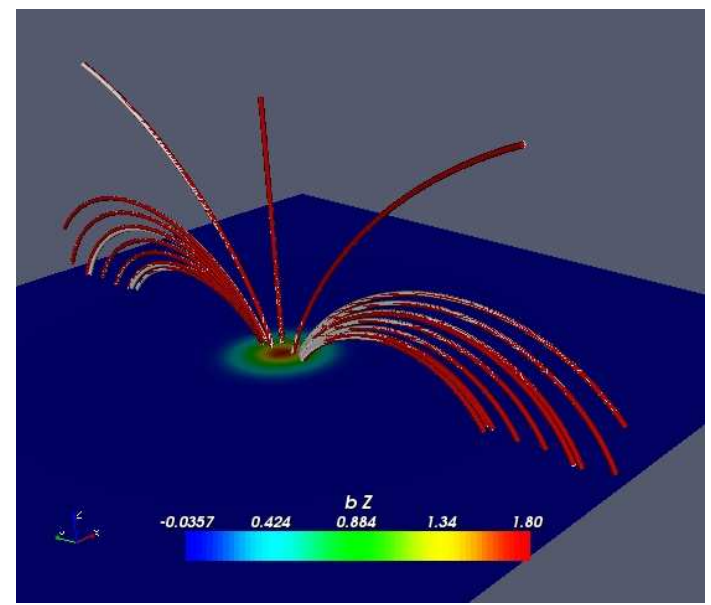

FIG. 5.1. Magnetic fieldlines of the exact solution (in white) and the numerical solution (in red) for the dipole test.

and corresponds to the current density

$$
\boldsymbol{j}=-\frac{1}{r^{2}}\left(r^{2} \psi^{\prime}\right)^{\prime}(r)(\boldsymbol{x} \wedge \boldsymbol{a})-2 \frac{\psi^{\prime}(r)}{r}(\boldsymbol{x} \wedge \boldsymbol{a}) .
$$

Here $r=\left(x_{1}^{2}+x_{2}^{2}+x_{3}^{2}\right), \boldsymbol{a}=(-2,3,1)$ and

$$
\psi(r)=(r-1)(r-2) .
$$

One can observe that the $L^{2}$-error on the curl $\boldsymbol{b}$ decreases linearly with $h$, while the

\begin{tabular}{|c|c|c|c|}
\hline \multirow{2}{*}{$h$} & \multirow{2}{*}{ DOF (edges) } & $\mid \operatorname{curl} \boldsymbol{b}-\operatorname{curl} \boldsymbol{u}_{h} \|_{0, \Omega}$ & $\left\|\boldsymbol{b}-\boldsymbol{u}_{h}\right\|_{L^{2}}$ \\
\hline & & $\|\operatorname{curl} \boldsymbol{b}\|_{0, \Omega}$ & $\|\boldsymbol{b}\|_{L^{2}}$ \\
\hline 0.5076 & 15012 & 0.0727 & 0.0707 \\
\hline 0.4108 & 29802 & 0.0580 & 0.0489 \\
\hline 0.3454 & 52032 & 0.0482 & 0.0360 \\
\hline 0.2622 & 124860 & 0.0361 & 0.0217 \\
\hline 0.2116 & 245592 & 0.0289 & 0.0149 \\
\hline
\end{tabular}
$L^{2}$ error on $\boldsymbol{b}$ decreases as $h^{1.8}$.

The div-curl problem on $\boldsymbol{b}$ : the relative $L^{2}$ and $H(\mathbf{c u r l})$ errors obtained with Nédelec' edge elements of degree 1 .

Preliminary test 3: the transport equation on $\lambda$.. In this test, $\Omega=[0,1] \times$ $[0,1] \times[0,1]$. In Table 5.3 we display the $L^{\infty}$ and the $L^{2}$ errors between the exact solution of the hyperbolic problem (4.19) and the approximated one. The former is given by

$$
\lambda(\boldsymbol{x})=x y z,
$$

Observe that $\lambda$ is solution to (4.19) with

$$
\boldsymbol{B}=\left(\begin{array}{c}
x \\
y \\
-2 z
\end{array}\right) \text { and } f=0 .
$$


We choose in this test $\sigma=0.0001$ and $\delta_{h}=0.1 h$.

\begin{tabular}{ccccc}
\hline$h$ & DOF (nodes) & $\frac{\left\|\lambda-\lambda_{h}\right\|_{\infty}}{\|\lambda\|_{\infty}}$ & & $\frac{\left\|\lambda-\lambda_{h}\right\|_{L^{2}}}{\|\lambda\|_{L^{2}}}$ \\
\hline 0.34641 & 216 & 0.03116 & 0.01994 \\
0.17322 & 1331 & 0.01163 & 0.00459 \\
0.08660 & 9261 & 0.00407 & 0.00109 \\
0.05773 & 29791 & 0.00175 & 0.00048 \\
0.04330 & 68921 & 0.00088 & 0.00027 \\
\hline \multicolumn{3}{c}{ TABLE 5.3}
\end{tabular}

The hyperbolic problem on $\lambda$ : the relative $L^{2}$ and $L^{\infty}$ errors obtained by the use of streamline diffusion method and $P^{1}$ conforming elements.

Notice that both the errors are decreasing with $h$. Nevertheless, a simple investigation of these numerical results shows that $L^{2}$ error decreases as $h^{2.06}$ while the $L^{\infty}$ error is decreasing as $h^{1.5}$.

5.2. The convergence of the iterative algorithm. Our task now is to check convergence of the iterative algorithm exposed in Section 3. The experience is realized in the domain $\Omega=B_{2}-\bar{B}_{1}$. We also compare the approximated pair $\left(\boldsymbol{B}_{h}, \lambda_{h}\right)$ obtained after convergence of the process with the exact solution $(\boldsymbol{B}, \lambda)$. The latter is given by:

$$
\boldsymbol{B}(\boldsymbol{x})=\frac{1}{\sqrt{\rho}}\left(\boldsymbol{e}_{\theta}+\boldsymbol{e}_{z}\right), \lambda(\boldsymbol{x})=\frac{1}{2 \rho} .
$$

This is a cylindrical Beltrami flow obtained by choosing $\eta(t)=t / 2$ (see Example 2 in the introduction). Here $(\rho, \theta, z)$ denote the cylindrical coordinates with respect to the point $(-3,-2,0)(\rho=0$ means the vertical axis $(-3,-2, z), z \in \mathbb{R})$.

Table 5.4 shows the rates of convergence of the sequence $\left(\boldsymbol{B}_{h}^{(n)}, \lambda_{h}^{(n)}\right)$ and the $L^{\infty}$ norm of $\operatorname{curl} \boldsymbol{B}_{h}^{(n)} \times \boldsymbol{B}_{h}^{(n)}$ versus $n$, the number of iterations. In Table 5.5, the error between the computed Beltrami flow $\left(\boldsymbol{B}_{h}, \lambda_{h}\right)$, obtained after convergence of the process, and the exact one is displayed. Observe the following points. On one hand, the iterative algorithm seems to be super-convergent since the sequence $\left(\boldsymbol{B}_{h}^{(n)}, \lambda_{h}^{(n)}\right)$ reaches the approximated solution $\left(\boldsymbol{B}_{h}, \lambda_{h}\right)$ after few iterations. On the other hand, this example shows that the convergence seems to be indeed achieved also for a domain with a non-connected boundary, even if the theory is not assuring this fact. Notice also that the error between the approximated pair $\left(\boldsymbol{B}_{h}, \lambda_{h}\right)$ and the exact one decreases strongly with $h$. Indeed, Table 5.5 shows that the errors $\left\|\boldsymbol{B}_{h}-\boldsymbol{B}\right\|_{H(\mathbf{c u r l})},\left\|\boldsymbol{B}_{h}-\boldsymbol{B}\right\|_{L^{2}}$ and $\|\lambda-\lambda\|_{\infty}$ decrease as $h, h^{2}$ and $h^{1.5}$ respectively. All these observations indicate the efficiency of the method and its actual performance.

Let us remark that the field $\boldsymbol{B}_{h}$ obtained after convergence remains force-free when the function $\lambda_{0}$ is slightly modified while $g$ is maintained.

5.3. Numerical properties of force-free fields. In this paragraph, the emphasis is on some properties of non linear Beltrami flows. $\Omega$ is again chosen as the domain $\Omega=B_{2}-B_{1}$. We are in particular interested in the behavior of the energy and the relative helicity when the flow moves away from the irrotational one. Both of these quantities are computed numerically. Recall that the energy of a vector field $\boldsymbol{B}$ 


\begin{tabular}{cccc}
\hline \multirow{2}{*}{ Iteration (n) } & $\left\|\boldsymbol{B}_{h}^{(n+1)}-\boldsymbol{B}_{h}^{(n)}\right\|_{H(\text { curl })}$ & $\frac{\left\|\lambda_{h}^{(n+1)}-\lambda_{h}^{(n)}\right\|_{\infty}}{\left\|\boldsymbol{B}_{h}^{(n)}\right\|_{H(\text { curl })}}$ & $\mid$ curl $\boldsymbol{B}_{h}^{(n)} \times\left.\boldsymbol{B}_{h}^{(n)}\right|_{\infty}$ \\
\hline 1 & 0.13695 & - & $1.3 \mathrm{e}-14$ \\
2 & 0.00764 & 0.02490 & 0.01217 \\
3 & 0.00022 & 0.00026 & 0.00677 \\
4 & $8.13 \mathrm{e}-06$ & $6.0 \mathrm{e}-05$ & 0.00676 \\
5 & $1.42 \mathrm{e}-06$ & 0. & 0.00676 \\
\hline
\end{tabular}

The residual difference $\|\left(\boldsymbol{B}_{h}^{(n+1)}, \lambda_{h}^{\text {TABLE }} 5.4-\left(\boldsymbol{B}_{h}^{(n)}, \lambda_{h}^{(n)}\right) \|_{H(\mathbf{c u r l}) \times L^{\infty}}\right.$ and the norm $\left|\operatorname{curl} \boldsymbol{B}_{h}^{(n)} \times \boldsymbol{B}_{h}^{(n)}\right|_{\infty}$ versus the number of iterations $n$. The strong decay of the residue indicates that the iterative process is super-convergent.

\begin{tabular}{cccc}
\hline$h$ & $\frac{\| \text { curl } \boldsymbol{B}-\mathbf{c u r l} \boldsymbol{B}_{h} \|_{0, \Omega}}{\|\operatorname{curl} \boldsymbol{B}\|_{0, \Omega}}$ & $\frac{\left\|\boldsymbol{B}-\boldsymbol{B}_{h}\right\|_{L^{2}}}{\|\boldsymbol{B}\|_{L^{2}}}$ & $\frac{\left\|\lambda-\lambda_{h}\right\|_{\infty}}{\|\lambda\|_{\infty}}$ \\
\hline 0.5459 & 0.0461 & 0.00988 & 0.00543 \\
0.3732 & 0.0297 & 0.00426 & 0.00356 \\
0.2839 & 0.0219 & 0.00237 & 0.00197 \\
0.2536 & 0.0194 & 0.00187 & 0.00162 \\
0.2293 & 0.0174 & 0.00150 & 0.00134 \\
\hline \multicolumn{4}{r}{ TABLE 5.5 }
\end{tabular}

The $H(\mathbf{c u r l})$ and $L^{2}$ errors on $\boldsymbol{B}$ and the $L^{\infty}$ error on $\lambda$ versus the discretization $h$.

is defined as

$$
E(\boldsymbol{B})=\int_{\Omega}|\boldsymbol{B}|^{2} d \boldsymbol{x} .
$$

We have

$$
E(\boldsymbol{B})=E_{0}+E(\boldsymbol{b})
$$

Here $E_{0}=E\left(\boldsymbol{B}_{0}\right)$ and $\boldsymbol{b}=\boldsymbol{B}-\boldsymbol{B}_{0}$ where $\boldsymbol{B}_{0}$ stands for the irrotational vector field corresponding to $g$, given by $\boldsymbol{B}_{0}=\nabla \chi$ with

$$
\Delta \chi=0 \text { in } \Omega, \frac{\partial \chi}{\partial n}=g \text { on } \partial \Omega .
$$

The relative helicity of $\boldsymbol{B}$ is defined as

$$
H(\boldsymbol{B})=\int_{\Omega} \boldsymbol{A} \cdot \boldsymbol{B} d x-\int_{\Omega} \boldsymbol{A}_{0} \cdot \boldsymbol{B}_{0} d x
$$

where $\boldsymbol{A}$ is a vector potential of $\boldsymbol{B}$ (i. e., $\operatorname{curl} \boldsymbol{A}=\boldsymbol{B}), \boldsymbol{B}_{0}$ is the potential field associated to $\boldsymbol{B}$ (i. e. $\boldsymbol{c u r l} \boldsymbol{B}_{0}=\mathbf{0}, \operatorname{div} \boldsymbol{B}_{0}=0$ and $\boldsymbol{B}_{0} . \boldsymbol{n}=\boldsymbol{B} . \boldsymbol{n}$ on $\partial \Omega$ ) and $\boldsymbol{A}_{0}$ is solution to the system

$$
\left\{\begin{array}{l}
\text { curlcurl } \boldsymbol{A}_{0}=0 \text { in } \Omega, \operatorname{div} \boldsymbol{A}_{0}=0 \text { in } \Omega, \\
\boldsymbol{A}_{0} \times \boldsymbol{n}=\boldsymbol{A} \times \boldsymbol{n} \text { on } \partial \Omega, \\
\int_{\Gamma} \boldsymbol{A}_{0} \cdot \boldsymbol{n} d \sigma=\int_{\Gamma_{i}} \boldsymbol{A} \cdot \boldsymbol{n} d \sigma, \text { for all } i=0, \ldots, p,
\end{array}\right.
$$

where $\Gamma_{0}, \ldots, \Gamma_{p}$ are the connected components of $\partial \Omega$. The relative helicity $H$ is gauge invariant and describes the linkage of the fieldlines of $\boldsymbol{B}$ with one another (see [12]). 
It is also an invariant of any perfect MHD motion of the plasma (see, e. g., [49] and [48]). Observe that the relative helicity of an irrotational field is zero.

Another point worth noting is that $\operatorname{curl} \boldsymbol{A}_{0}=\boldsymbol{B}_{0}$ since

$$
\operatorname{curl} \boldsymbol{A}_{0} . \boldsymbol{n}=\operatorname{div}_{T}\left(\boldsymbol{A}_{0} \times \boldsymbol{n}\right)=\operatorname{curl} \boldsymbol{A} . \boldsymbol{n}=\boldsymbol{B}_{0} . \boldsymbol{n} .
$$

Moreover, choosing $\boldsymbol{A}$ such that $\boldsymbol{A} \cdot \boldsymbol{n}=0$ on $\partial \Omega$ and introducing the vector function $\boldsymbol{A}_{0}^{*}$ defined as the unique element of $U$ satisfying $\operatorname{curl} \boldsymbol{A}_{0}^{*}=\boldsymbol{B}_{0}$ and $\operatorname{div} \boldsymbol{A}_{0}^{*}=0$, we get

$$
H(\boldsymbol{B})=\int_{\Omega} \boldsymbol{b} \cdot\left(\boldsymbol{A}+\boldsymbol{A}_{0}^{*}\right) d x
$$

Actually,

$$
\begin{aligned}
H(B) & =\int_{\Omega}\left(\boldsymbol{A}-\boldsymbol{A}_{0}\right) \cdot \boldsymbol{B}+\int_{\Omega} \boldsymbol{A}_{0} \cdot \boldsymbol{b} d x \\
& =\int_{\Omega}\left(\boldsymbol{A}-\boldsymbol{A}_{0}\right) \cdot \boldsymbol{B}+\int_{\Omega} \boldsymbol{A}_{0} \cdot \operatorname{curl}\left(\boldsymbol{A}-\boldsymbol{A}_{0}\right) d x \\
& =\int_{\Omega}\left(\boldsymbol{A}-\boldsymbol{A}_{0}\right) \cdot\left(\boldsymbol{B}+\boldsymbol{B}_{0}\right) d x \\
& =\int_{\Omega}\left(\boldsymbol{A}-\boldsymbol{A}_{0}\right) \cdot \operatorname{curl}\left(\boldsymbol{A}+\boldsymbol{A}_{0}^{*}\right) d x,
\end{aligned}
$$

and (5.3) is easily obtained after using Stokes formula. Now, let us give some estimates of $E(\boldsymbol{B})$ and $H(\boldsymbol{B})$ in terms of the $E_{0}$, the energy of the potential field $\boldsymbol{B}_{0}$. Firstly, observe that

$$
E(\boldsymbol{b})=\|\boldsymbol{b}\|_{L^{2}}^{2} \leq \mu_{0}^{-2}\|\operatorname{curl} \boldsymbol{b}\|_{L^{2}}^{2}=\mu_{0}^{-2}\|\lambda \boldsymbol{B}\|_{L^{2}}^{2} \leq \frac{\left\|\alpha_{0}\right\|_{\infty}^{2}}{\mu_{0}^{2}}\|\boldsymbol{B}\|_{L^{2}}^{2} .
$$

Thus,

$$
E(\boldsymbol{b}) \leq s^{2} E(\boldsymbol{B})
$$

where

$$
s=\frac{\left\|\alpha_{0}\right\|_{\infty}}{\mu_{0}} .
$$

Combining with identity (5.2) gives

$$
E_{0} \leq E(\boldsymbol{B}) \leq E_{\max }=\tau E_{0},
$$

where

$$
\tau=\frac{1}{1-s^{2}}
$$

On the other hand, choosing $\boldsymbol{A} \in U$ and using (5.3) yields

$$
H(\boldsymbol{B})^{2} \leq\|\boldsymbol{b}\|_{L^{2}(\Omega)}^{2}\left\|\boldsymbol{A}+\boldsymbol{A}_{0}^{*}\right\|_{L^{2}(\Omega)}^{2} .
$$

Combining with the inequality

$$
\left\|\boldsymbol{A}+\boldsymbol{A}_{0}^{*}\right\|_{L^{2}(\Omega)} \leq \mu_{0}^{-1}\left\|\operatorname{curl}\left(\boldsymbol{A}+\boldsymbol{A}_{0}^{*}\right)\right\|_{L^{2}(\Omega)}=\mu_{0}^{-1}\left\|\boldsymbol{B}+\boldsymbol{B}_{0}\right\|_{L^{2}(\Omega)} .
$$


gives

$$
H(\boldsymbol{B})^{2} \leq \mu_{0}^{-2}\|\boldsymbol{b}\|_{L^{2}(\Omega)}^{2}\left\|\boldsymbol{B}+\boldsymbol{B}_{0}\right\|_{L^{2}(\Omega)}^{2}
$$

(note that $\int_{\Omega} \boldsymbol{b} . \boldsymbol{B}_{0} d x=0$ ) Since,

$$
\left\|\boldsymbol{B}+\boldsymbol{B}_{0}\right\|_{L^{2}}^{2}=\left\|\boldsymbol{b}+2 \boldsymbol{B}_{0}\right\|_{L^{2}}^{2}=\|\boldsymbol{b}\|_{L^{2}}^{2}+4\left\|\boldsymbol{B}_{0}\right\|_{L^{2}}^{2} .
$$

we deduce that

$$
H(\boldsymbol{B})^{2} \leq \mu_{0}^{-2} E(\boldsymbol{b})\left(E(\boldsymbol{b})+4 E_{0}\right) .
$$

Using (5.4) and (5.5) leads to

$$
H(\boldsymbol{B})^{2} \leq \mu_{0}^{-2} \frac{s^{2}\left(4-3 s^{2}\right)}{\left(1-s^{2}\right)^{2}} E_{0}^{2}=\mu_{0}^{-2}(\tau-1)(\tau+3) E_{0}^{2} .
$$

Finally, we get

$$
|H(\boldsymbol{B})| \leq H_{\max }
$$

with

$$
H_{\max }=(\tau+3)^{1 / 2}(\tau-1)^{1 / 2} \frac{E_{0}}{\mu_{0}} .
$$

Estimates (5.7) suggests that energy and relative helicity of a Beltrami field $\boldsymbol{B}$ increase with $\lambda$. This fact will be confirmed by the forthcoming numerical results.

For the numerical tests, the normal component $\boldsymbol{B} . \boldsymbol{n}=g$ is fixed and is given by $g=\boldsymbol{B}_{\text {dip }} . \boldsymbol{n}$, where $\boldsymbol{B}_{\text {dip }}$ is the dipolar field defined as

$$
\boldsymbol{B}_{\text {dip }}=\frac{B_{0}}{r^{3}}\left(2 \cos (\theta) \boldsymbol{e}_{r}+\sin (\theta) \boldsymbol{e}_{\theta}\right) .
$$

Here $(r, \theta, \phi)$ denotes the spherical coordinates.

The function $\alpha_{0}(\boldsymbol{x})$ is taken into the form

$$
\alpha_{0}(\boldsymbol{x})=\beta \lambda_{00}(\boldsymbol{x}),
$$

where $\beta \in \mathbb{R}$ is a free parameter which describes the strength of $\lambda(\boldsymbol{x})$, and $\lambda_{00}(\boldsymbol{x})$ is such that

$$
\left|\lambda_{00}\right|_{L^{\infty}\left(\Sigma^{-}\right)}=1
$$

More precisely, we choose

$$
\lambda_{00}(\boldsymbol{x})=\exp ^{-\left(\theta-\frac{\pi}{2}\right)^{2}} .
$$

Note that $\alpha_{0}$ is equal to $\beta$ on the equator of the interior sphere and is minimal at the poles.

We are also interested in comparing the non-linear Beltrami field $\boldsymbol{B}$ with the linear force-free field $\boldsymbol{B}_{l}$ solution of

$$
\operatorname{curl} \boldsymbol{B}_{l}=\beta \boldsymbol{B}_{l} \text { in } \Omega, \operatorname{div} \boldsymbol{B}_{l}=0 \text { in } \Omega ; \boldsymbol{B}_{l} . \boldsymbol{n}=g \text { on } \partial \Omega .
$$

This problem is also solved using the iterative algorithm of Section 3 and $H$ (curl) elements. The reaction-convection problem on $\lambda$ is dropped since $\lambda$ is known in this last case. Table 5.6 contains the values of the quantities $H(\boldsymbol{B}), E(\boldsymbol{B}), H\left(\boldsymbol{B}_{l}\right), E\left(\boldsymbol{B}_{l}\right)$ for several values of $\beta$.

A lesson can be drawn from Table 5.6: the energy, the helicity and the complexity of the topology of a Beltrami field increase with the amplitude of its parameter $\lambda$. 


\begin{tabular}{ccccc}
$\beta$ & $H(\boldsymbol{B})$ & $H\left(\boldsymbol{B}_{l}\right)$ & $\frac{E(\boldsymbol{B})}{E_{0}}$ & $\frac{E\left(\boldsymbol{B}_{l}\right)}{E_{0}}$ \\
\hline 0 & 0 & 0 & 1 & 1 \\
1 & 2.21 & 10.30 & 1.006 & 1.088 \\
2 & 4.60 & 30.51 & 1.025 & 1.611 \\
2.5 & 5.90 & 60.77 & 1.041 & 2.744 \\
3 & 7.33 & 210.08 & 1.063 & 9.660 \\
\hline \multicolumn{5}{c}{ TABLE 5.6 }
\end{tabular}

The helicity and the energy of the linear and non-linear force-free fields $\boldsymbol{B}$ and $\boldsymbol{B}_{l}$ for several values. Notice that these quantities increase in both cases with $\beta$.

5.4. Cylindrical test. Here we propose to compute force free fields in cylindrical geometries. Denote $(\rho, \theta, z)$ the cylindrical coordinates (relative to the origin). We consider the force free field $\boldsymbol{B}$ defined by

$$
\boldsymbol{B}=\left(\begin{array}{c}
B_{\rho} \\
B_{\theta} \\
B_{z}
\end{array}\right)=\left(\begin{array}{c}
0 \\
-\frac{\eta_{0}(\rho) \rho}{1+\rho^{2} \eta_{0}(\rho)^{2}} c(\rho) \\
\frac{1}{1+\eta_{0}(\rho)^{2} \rho^{2}} c(\rho)
\end{array}\right)
$$

where $\eta_{0}(\rho)$ is a given function depending only on $\rho$, and $c(\rho)$ is defined by

$$
c(\rho)=C_{0} \exp \left(\int \frac{\rho^{2} \eta_{0}(\rho) \eta_{0}^{\prime}(\rho)}{1+\rho^{2} \eta_{0}(\rho)^{2}}\right)
$$

with $C_{0} \in \mathbb{R}$. $\lambda$ is defined by

$$
\lambda=-\frac{2 \eta_{0}(\rho)+\eta_{0}^{\prime}(\rho) \rho}{1+\rho^{2} \eta_{0}(\rho)^{2}} .
$$

Magnetic fieldlines live on isosurfaces of $\lambda$ which are nothing else than cylinders. Here, we choose $\eta_{0}(\rho)$ of the form

$$
\eta_{0}(\rho)=\rho^{r}, \quad r \in \mathbb{N} .
$$

In that case,

$$
\boldsymbol{B}=\left(\begin{array}{c}
0 \\
-\frac{\rho^{r+1}}{\left(1+\rho^{2 r+2}\right)^{\frac{r+2}{2 r+2}}} \\
\frac{1}{\left(1+\rho^{2 r+2}\right)^{\frac{r+2}{2 r+2}}}
\end{array}\right)
$$

and

$$
\lambda=-\frac{(2+r) \rho^{r}}{1+\rho^{r+2}}
$$

We show numerical solution obtained in the cylinder of diameter equal to $\rho \max =1.6$ and of height $Z_{\max }=4$. The size of the mesh is $h=0.120343$ with 124416 elements. 


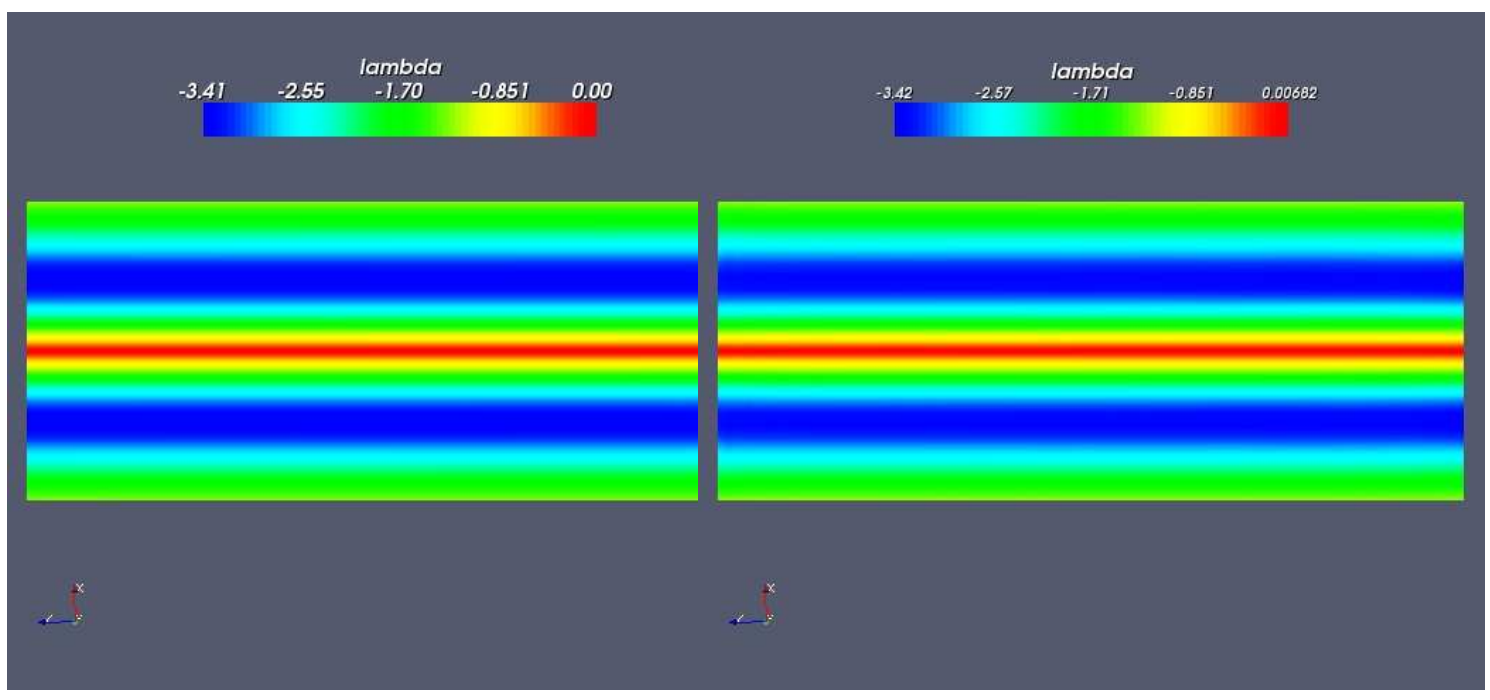

Fig. 5.2. Profile of $\lambda$ on $\Omega \cap\{y=0\}$. The exact solution is presented on the left and the numerical one on the right.

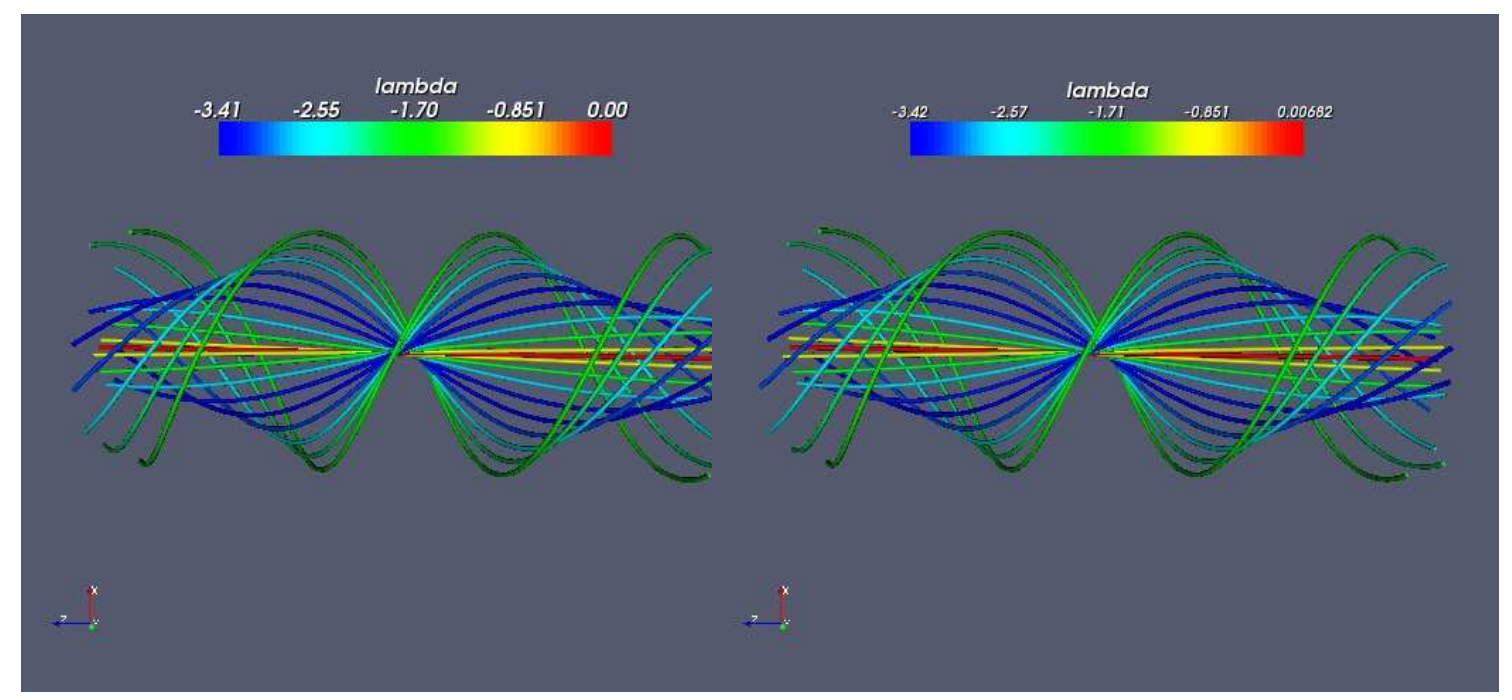
by $\lambda$

FIG. 5.3. Visualisation of fieldlines of the exact (left) and the numerical (right) solution colored

The relative error in norm $L^{2}$ on $\boldsymbol{B}$ between the exact solution and the numerical one is equal to 0.01623 . The relative error on $\lambda$ is equal to $7.43 .10^{-3}$. In Figures 5.2 we display a cut at $y=0$ of the profile of $\lambda$ for the exact and the numerical solutions. The fieldlines are shown in Figures 5.3. In Figures 5.4, we visualize fieldlines enclosing the cylindrical isosurface on which $\lambda=-2.9$.

We propose also to compare this non-linear FFF with some linear ones, satisfying the same boundary conditions. In Fig 5.5, we display a potential field (or a linear force-free field with $\lambda=0$ ) and a linear force-free field with $\lambda=1$. In Fig 5.6, we present an other linear force-free field with $\lambda=3$ and the non-linear force-free field 


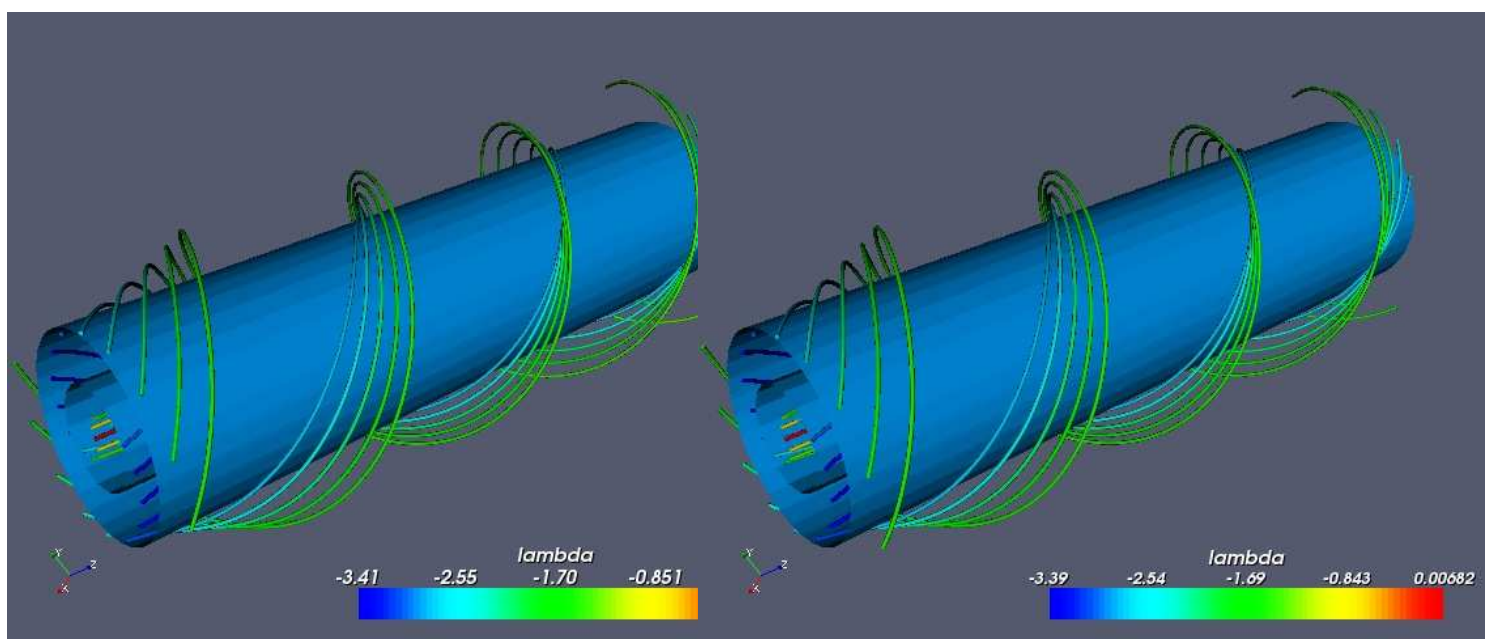

FIG. 5.4. Presentation of fieldlines enclosing an isosurface which is nothing else than a cylinder. We choose to represent the isosurface corresponding to $\lambda=-2.9$.

given above. One can observe the influence of the value of $\lambda$ on the topology of magnetic fieldlines comparing the cases $\lambda=0,1,3$. The complexity of topology of fieldlines increase with lambda.

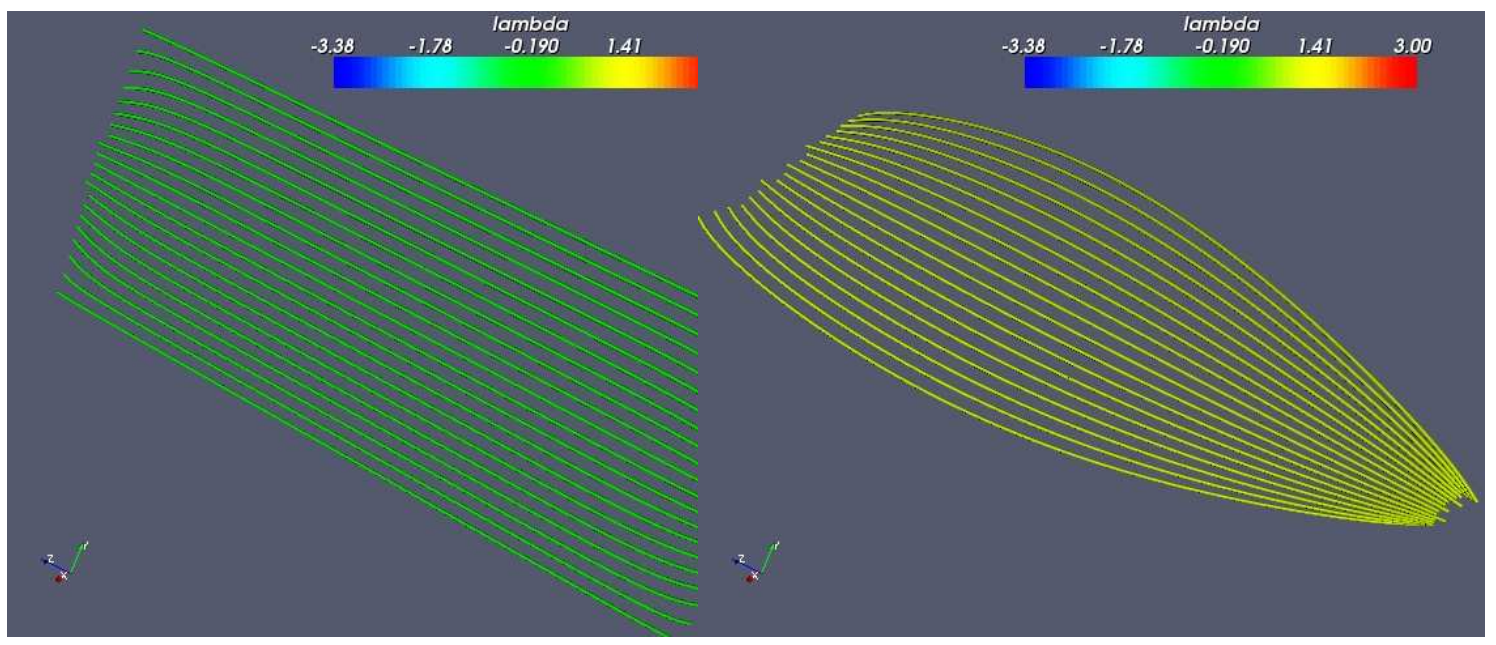

FIG. 5.5. Potential field and (on the left) and linear force-free field in the case $\lambda=1$ (on the right).

5.5. Beltrami field in a half torus. In termonuclear fusion, equilibrium are described by the full magnetostatic system. The $\beta$ number referring to the ratio of the plasma pressure over the magnetic pressure is generally small. In order to show the efficiency of the method on more complicated cases and in view to extend our method to the full magnetostatic system, we propose to test our 3D code on force-free configurations comparing partially our results with pressureless solutions obtained by the 2D axisymmetric code CHEASE developed by H. Lütjens. and al. (see [43]).

In this test, the domain $\Omega$ corresponds to a half torus and is subdivided into 428064 


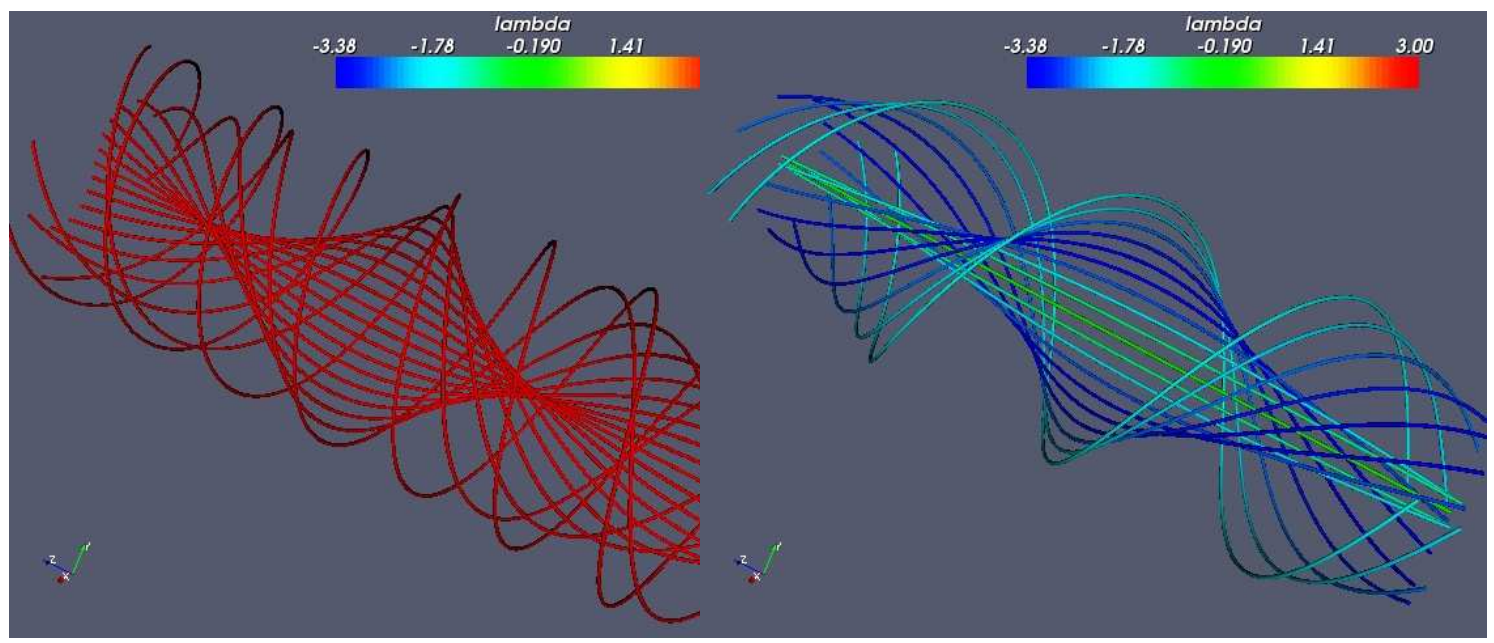

FIG. 5.6. Linear force-free field in the case $\lambda=3$ (on the left) and non-linear force-free field (on the right) described before.

elements with a mesh size equal to $h=0.0389541$. The normal component $g$ of the magnetic field is given at the two poloidal sections $S_{i}, i=1,2$ of this half-torus and on the lateral boundary $\partial \Omega \backslash S_{1} \cup S_{2}$, with

$$
g<0 \text { on } S_{1}, g>0 \text { on } S_{2} \text { and } g=0 \text { on } \partial \Omega \backslash S_{1} \cup S_{2} .
$$

The function $\alpha_{0}$ is given on the inflow section $S_{1}$. These quantities $g$ and $\alpha_{0}$ are computed by the code CHEASE in the whole torus and then used as data for TETRAFFF.

The relative difference on $\boldsymbol{B}$ between the CHEASE solution and our numerical solution in norm $L^{2}$ is equal to 0.00918812 .

In Figure 5.7 we display fieldlines obtained with the CHEASE code and with our code. Figure 5.8 shows an isosurface $(\lambda=1.25)$ on which lie magnetic fieldlines. In Tokamak, those surfaces are called magnetic surfaces. In Figure 5.9, we present a cut magnetic surfaces corresponding to constant values of $\lambda$. This illustrate the well known property of Tokamak plasmas whose magnetic surfaces form stacked toric surfaces.

6. Comments. The numerous numerical tests we have done show the quick convergence of the algorithm, although the proof of this convergence from a theorical view point remains an unsolved question. However, as conjectured in section 3, we can verify numerically that convergence can be lost for boundary data on $\lambda$ too large. In spite of these limits, the algorithm allows to test a very great number of interesting cases.

Our next objective consists to extend our software to multiply-connected geometries. Another perspective of this work is to extend this method to solve the full magnetostatic equations in order to apply the algorithm in fusion problem, and particularly in tokamaks. This will be done in a forthcoming paper.

\section{Appendix A.}

The existence and the unicity of a solution to the problem (4.2) can be proved by 


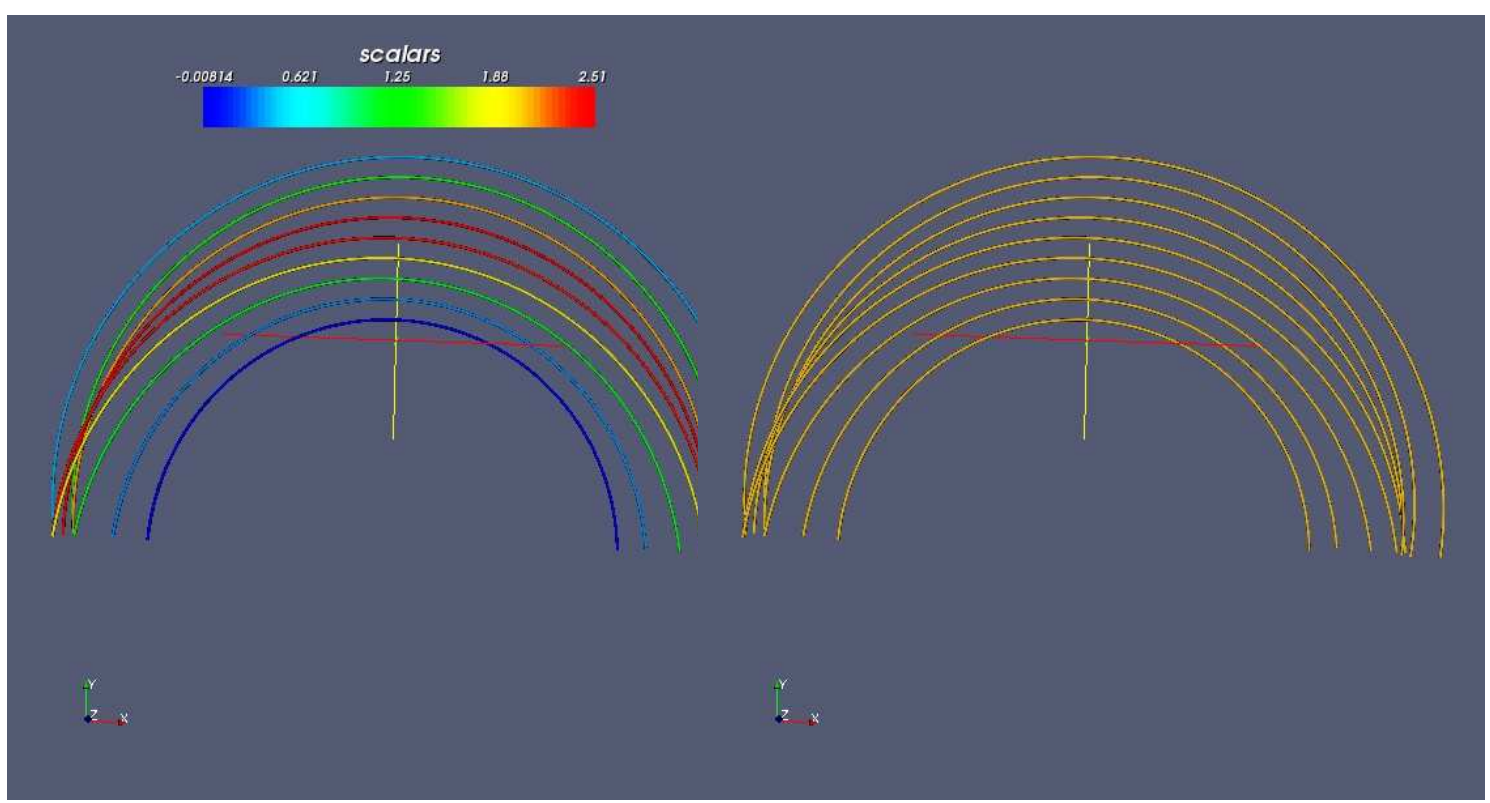

FIG. 5.7. Equilibria obtained by CHEASE on the right and our code on the left.

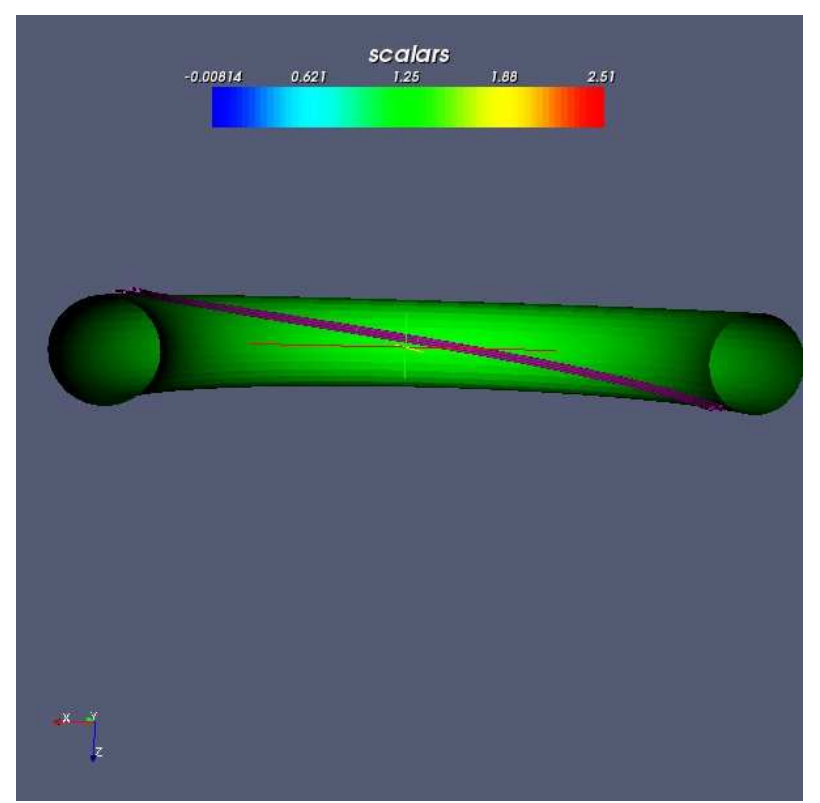

FIG. 5.8. Field lines around a magnetic surface.

mean of Babuska-Brezzi theorem. Consider the subspace $V$ of $W^{0}$ defined as

$$
\begin{aligned}
V & =\left\{\boldsymbol{p} \in W^{0}(\Omega), \quad \int_{\Omega} v \operatorname{div} \boldsymbol{p} d x=0, \forall v \in L_{0}^{2}(\Omega)\right\}, \\
& =\left\{\boldsymbol{p} \in W^{0}(\Omega), \quad \operatorname{div} \boldsymbol{p}=c t e\right\}, \\
& =\left\{\boldsymbol{p} \in W^{0}(\Omega), \quad \operatorname{div} \boldsymbol{p}=0\right\} .
\end{aligned}
$$




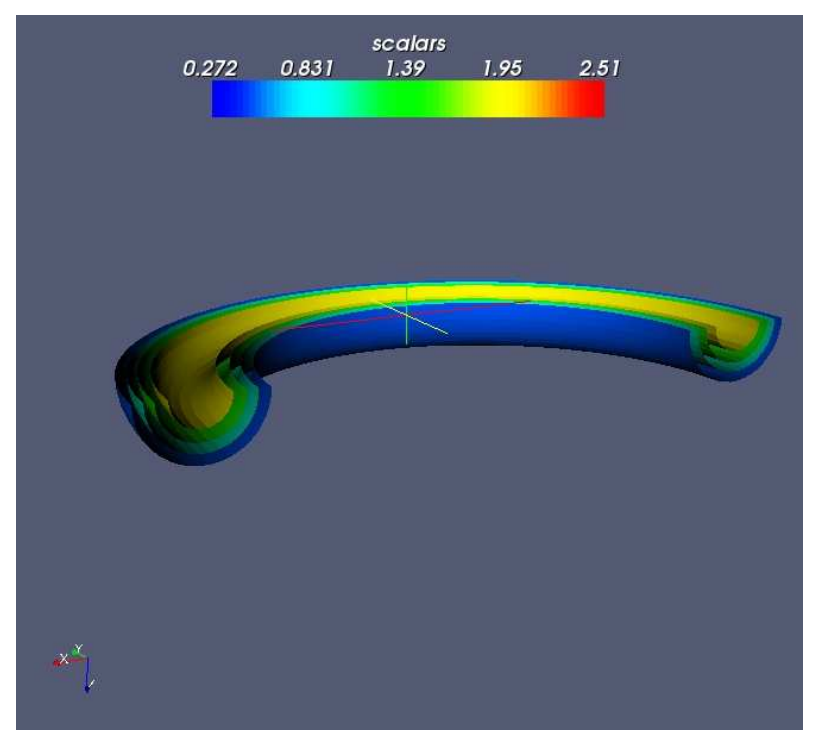

FIG. 5.9. Cut of the stacked toric magnetic surfaces.

It is quite clear that the continuous bilinear form defined on $W^{0}(\Omega) \times W^{0}(\Omega)$ by

$$
a(\boldsymbol{p}, \boldsymbol{q})=\int_{\Omega} \boldsymbol{p} . \boldsymbol{q},(\boldsymbol{p}, \boldsymbol{q}) \in W^{0}(\Omega) \times W^{0}(\Omega)
$$

is V-elliptic.

Consider the continuous bilinear form defined on $W^{0}(\Omega) \times L_{0}^{2}(\Omega)$ by

$$
b(\boldsymbol{q}, u)=\int_{\Omega} u \operatorname{div} \boldsymbol{q}
$$

with $\boldsymbol{q} \in W^{0}$ and $u \in L_{0}^{2}(\Omega)$.

We can prove that the inf-sup condition is statisfied for $b(.,$.$) . Let us introduce the$ map $\mathcal{R}$ defined on $L_{0}^{2}(\Omega)$ by

$$
\mathcal{R} v=\nabla \phi_{v}, \quad \forall v \in L_{0}^{2}(\Omega)
$$

where $\phi_{v} \in H^{1}(\Omega)$ with $\int_{\Omega} \phi_{v}=0$ is solution of

$$
\left\{\begin{array}{l}
\Delta \phi_{v}=v \text { dans } \Omega \\
\frac{\partial \phi_{v}}{\partial n}=0 \text { sur } \partial \Omega .
\end{array}\right.
$$

As $b(\mathcal{R} v, v)=\|v\|_{0, \Omega}^{2}$, we have

$$
\begin{aligned}
\inf _{\|v\|_{0, \Omega}=1} \sup _{\|q\|_{H(\text { div } ; \Omega)}=1} b(\boldsymbol{q}, v) & =\inf _{\boldsymbol{v} \neq 0} \sup _{\boldsymbol{q} \neq 0} \frac{b(\boldsymbol{q}, v)}{\left.\|\boldsymbol{q}\|_{H(\text { div }} ; \Omega\right)} \\
& \geq \inf _{v \neq 0} \frac{b(\mathcal{R} v, v)}{\|\mathcal{R} v\|_{H(\text { div } ; \Omega)}}=\inf _{v \neq 0} \frac{\|v\|_{0, \Omega}}{\|\mathcal{R} v\|_{H(\text { div } ; \Omega)}} \\
& \geq \frac{1}{\|\mathcal{R}\|}>0 .
\end{aligned}
$$


Appendix B. Existence and uniqueness are straightforward consequences of (4.21). It remains to prove the error estimate. We set $\xi=\lambda-\lambda_{h}$. Then,

$$
a_{h}(\xi, \xi)=a_{h}\left(\xi, \lambda-w_{h}\right) \text { for all } w_{h} \in W_{h} .
$$

Let $w_{h} \in W_{h}$ and set $\varphi=\lambda-w_{h}$. Then,

$$
\begin{aligned}
\left|a_{h}(\xi, \varphi)\right| \leq & \delta_{h}\|\boldsymbol{B} \cdot \nabla \xi\|_{L^{2}(\Omega)}\|\boldsymbol{B} \cdot \nabla \varphi\|_{L^{2}(\Omega)}+\sigma\|\xi\|_{L^{2}(\Omega)}\|\varphi\|_{L^{2}(\Omega)} \\
& +\delta_{h} \sigma\|\xi\|_{L^{2}(\Omega)}\|\boldsymbol{B} \cdot \nabla \varphi\|_{L^{2}(\Omega)}+\|\boldsymbol{B} . \nabla \xi\|_{L^{2}(\Omega)}\|\varphi\|_{L^{2}(\Omega)} \\
& +\|\xi\|_{L_{*}^{2}(\partial \Omega)}\|\varphi\|_{L_{*}^{2}(\partial \Omega)} \\
\leq & {\left[\sqrt{\delta_{h}}\|\boldsymbol{B} \cdot \nabla \varphi\|_{L^{2}(\Omega)}+\sqrt{\sigma}\|\varphi\|_{L^{2}(\Omega)}+\delta_{h} \sqrt{\sigma}\|\boldsymbol{B} \cdot \nabla \varphi\|_{L^{2}(\Omega)}\right.} \\
& \left.+\delta_{h}^{-1 / 2}\|\varphi\|_{L^{2}(\Omega)}+\|\varphi\|_{L_{*}^{2}(\partial \Omega)}\right]\|\xi\| \|_{\Omega} \\
\leq & K\left[\left(\sqrt{\delta_{h}}+\delta_{h} \sqrt{\sigma}\right)\|\boldsymbol{B}\|_{\infty} \cdot\|\varphi\|_{H^{1}(\Omega)}+\sqrt{\sigma}\|\varphi\|_{L^{2}(\Omega)}+\delta_{h}^{-1 / 2}\|\varphi\|_{L^{2}(\Omega)}\right. \\
& \left.+\delta_{h}^{-1 / 2}\|\varphi\|_{L^{2}(\Omega)}+\sqrt{\delta_{h}}\|\varphi\|_{H^{1}(\Omega)}\right]\|\xi\| \|_{\Omega},
\end{aligned}
$$

where we used the inequality (see, e. g., Grisvard [32], Th. 1.5.1.10)

$$
\|v\|_{L^{2}(\partial \Omega)}^{2} \leq K_{0}\left(\delta_{h}^{-1}\|v\|_{L^{2}(\Omega)}^{2}+\delta_{h}\|v\|_{H^{1}(\Omega)}^{2}\right)
$$

which is valid when $0<\delta_{h}<1$. The constant $K_{0}$ depends neither on $\delta_{h}$ nor on $v$. Thus, since $\delta_{h} \sigma<1$, we get

$$
\left(1-\sigma \delta_{h}\right)\left\||\xi \|| \leq C\left(\delta_{h}^{1 / 2}\|\varphi\|_{H^{1}(\Omega)}+\delta_{h}^{-1 / 2}\|\varphi\|_{L^{2}(\Omega)}\right) .\right.
$$

The proof is ended by using usual estimate on the interpolation error for conforming finite elements.

\section{REFERENCES}

[1] H.-D. AlBer, Existence of three-dimensional, steady, inviscid, incompressible flows with nonvanishing vorticity, Math. Ann. 292 (1992), no. 3, 493-528.

[2] T. Amari, J. J. Aly, J. F. Luciani, T. Z. Boulmezaoud and Z. Mikic, Reconstructing the solar coronal magnetic field as a force free magnetic field, Solar physics, 174 (1997), p. $129-149$.

[3] T. Amari , T. Z. Boulmezaoud And Y. Maday, A regularization method for the ill posed Cauchy problem encoutered in the Extrapolation of the photospheric magnetic field, Astronomy and Astrophysics, Vol. 339 (1998), p. 252-260.

[4] V. Agoshkov, Boundary value problems for transport equations. Modeling and Simulation in Science, Modeling and Simulation in Science, Engineering and Technology. Birkhäuser Boston, Inc., Boston, MA (1998).

[5] T. Amari and T. Z. Boulmezaoud and Z. Mikic, An iterative method for the reconstruction of the solar magnetic field, Astronomy and Astrophysics, Vol. 350, 1051-1059 (1999).

[6] C. Amrouche, C. Bernardi, M. Dauge and V. Girault, Vector potentials in threedimensional nonsmooth domains, Math. Methods Appl. Sci. 21, no. 9 (1998), p. 823-864.

[7] V. Arnold, Sur la topologie des écoulements stationnaires des fluides parfaits, C. R. Acad. Sci. 261 (Groupe 1) (1965), p. 17-20.

[8] P. Azerad, Analyse des équations de Navier-Stokes en bassin peu profond et de l'équation de transport, Ph. D. dessertation, Université de Neuchatel, Suisse (1996).

[9] I. BABuskA, The finite element method with Lagrangian multipliers, Numer. Math. 20 (19721973), p. 179-192.

[10] C. Bardos, Problèmes aux limites pour les équations aux dérivées partielles du premier ordre à coefficients réels, Ann. Scient. Ecol. Norm. Sup., 4ème série, T3 (1970), p. 185-233.

[11] A. Bendali and N. Raynaud and J.M. Thomas, New decomposition of shape functions spaces of mixed finite element methods, Appl. Math. Lett., 9 (1996), 33-38.

[12] M. A. Berger And G. B. Field, The topological properties of magnetic helicity, J. Fluid Mech. 147 (1984), p. 133-148. 
[13] M. Bineau, On the existence of force-free fields, Comm. Pure Appl. Math., Vol. 25, 77-84, (1972).

[14] T. Z. Boulmezaoud, On the existence of non-linear Beltrami fields, Comptes Rendus de l'Académie des Sciences, T. 328, Série I (1999), p. 437-442.

[15] T. Z. Boulmezaoud And T. Amari, On the existence of non-linear force-free fields in threedimensional multiply-connected domains, Zeitschrift für Angewandte Mathematik und Physik (ZAMP), 51, no. 6 (2000), p. 942-967.

[16] T. Z. Boulmezaoud And T. Amari, Approximation of linear force-free fields in bounded 3-D domains. Math. Comput. Modelling 31 (2000), no. 2-3, 109-129.

[17] T. Z. Boulmezaoud, Y. Maday and T. Amari, On the linear Beltrami fields in bounded and unbounded three-dimensional domains, Mathematical Modelling and Numerical Analysis, Vol. 33, No. 2 (1999), p. 359-394.

[18] F. BrEzzI, On the existence, uniqueness and approximation of saddle-point problems arising from Lagrangian multipliers, Rev. Française Automat. Informat. Recherche Opérationnelle Sér. Rouge, 8, R-2 (1974), p. 129-151.

[19] F. Brezzi, G. Hauke, L.D. Marini, G. Sangalli, Link-Cutting Bubbles for the Stabilization of Convection-Diffusion-Reaction Problems, Math. Models Methods Appl. Sci., Vol. 13 (3) (2003), p. 445-461.

[20] F. BREzZi And M. Fortin, A minimal stabilisation procedure for mixed finite element methods , Numer. Math., 89 (2001), 457-491.

[21] A. Brooks And T. R. J. Hughes, Streamline upwind/Petrov-Galerkin formulations for convection dominated flows with particular emphasis on the incompressible Navier-Stokes equations, FENOMECH '81, Part I (Stuttgart, 1981). Comput. Methods Appl. Mech. Engrg. 32 (1982), no. 1-3, 199-259.

[22] O. P. Bruno and P. Laurence, Existence of three-dimensional toroidal MHD equilibria with nonconstant pressure, Comm. Pure Appl. Math. 49 (1996), no. 7, p. 717-764

[23] S. Childress, New solutions of the kinematic dynamo problem, J. Mathematical Phys. 11, (1970) 3063-3076.

[24] P.-G. Ciarlet, The finite element method for elliptic problems, North-Holland, Amsterdam (1978).

[25] A. J. Chorin, A numerical method for solving incompressible viscous flow problems, J. Comput. Phys. 2, 12-26 (1967).

[26] T. Dombre, U. Frisch, J. M. Greene, M. Hénon, M., A. Mehr, A. M. Soward Chaotic streamlines in the ABC flows, J. Fluid Mech. 167 (1986), 353-391.

[27] J. P. Friedberg, Ideal Magnetohydrodynamics, New York: Plenum, 1987

[28] V. Girault and P. A. Raviart, Finite element methods for Navier-Stokes equations, Springer-Verlag, 1986.

[29] O. GLASS, Existence of solutions for the two-dimensional stationary Euler system for ideal fluids with arbitrary force, Ann. I. H. Poincaré, 6 (2003), p. 921-946.

[30] G. H. Golub And C. Greif, On solving block-structured indefinite linear systems, SIAM J. Sci. Comput. 24 (2003), no. 6, 2076-2092.

[31] G. H. Golub, C. Greif and J. M. Varah, An algebraic analysis of block diagonal preconditioner for saddle point systems, SIAM J. Matrix Anal. Appl. 27 (2005), no. 3, 779-792.

[32] P. GRISvard, Elliptic problems in nonsmooth domains, Pitman (1985).

[33] M. HÉnon, Sur la topologie des lignes de courant dans un cas particulier, C. R. Acad. Sci. 262 A (1966), p. 312-314.

[34] J.D Jachson, Classical Electrodynamics, John Wiley and Sons, 1995.

[35] C. Johnson, U. NÄvert and J. PitkÄranta, Finite element methods for linear hyperbolic problems, Comp. Meth. Appl. Mech. Engin., Vol. 45, 285-312 (1984).

[36] R. KRess, The treatement of a Neumann boundary value problem for force-free fields by an integral equation method, Proceedings of the Royal Society of Edimburgh, 82A (1978), p. 71-86.

[37] R. KRess, A remark on a boundary value problem for force-free fields, J. of Appl. Math. and Phys. (ZAMP), Vol. 28 (1977), p. 715-722.

[38] P. Laurence and M. Avellaneda, On Woltjer's variational principle for force-free fields, J. Math. Phys. 32, No. 5 (1991), p. 1240-1253.

[39] P. Lesaint, Sur la résolution des systèmes hyperboliques du premier ordre par des méthodes d'éléments finis, Thése de Doctorat, UPMC, Paris (1975).

[40] J.-Q. LI, J.-X. WANG AND F.-S. WEI, A fluid dynamics approach for the computation of nonlinear force-free fields, Chin. j. Astron. Astrophy., vol. 3, No. 3 (2003), p. 247-256.

[41] B. C. Low, Magnetic field configurations associated with polarity intrusion in a solar active region. I. the Force-free fields, Solar Physics 77 (1982), p. 43-61. 
[42] B. C. Low, Magnetic field configurations associated with polarity intrusion in a solar active region, Solar Physics 115 (1988), p. 269-276.

[43] H. Lütuens, A. Bondenson, And O. SAuter, The CHEASE code for toroidal MHD equilibria, Comput. Phys. Commun. 97 (1996), p. 219-267

[44] J. C. NÉDÉLEC, Mixed finite elements in $R^{3}$, Numer. Math. 35 (1980), no. 3, p. 315-341.

[45] E.N. PARKer, Cosmical Magnetic Fields. Their origin and their Activity, Clarendon Press: Oxford, 1979

[46] E. R. PRIEST, Solar Magnetohydrodynamics, Reidel, Dordrecht (1982).

[47] J.E. Roberts ans J.M. Thomas, Mixed and hybrid methods, Handbook of numerical analysis, Vol. II, (1991), p 523-639.

[48] J. B. TAYLOR, Relaxation of magnetic reconnection in plasmas, Rev. Mod. Phys. 58 (1986), p. $741-763$.

[49] L. Woltuer, A theorem on force-free magnetic fields, Proc. Nat. Acad. Sci. 44 (1958), p.489491. 